\title{
ARGONAUTE proteins regulate a specific network of genes through KLF4 in mouse embryonic stem cells
}

Madlen Müller ${ }^{1,2, £}$, Moritz Schaefer ${ }^{1,2, £}$, Tara Fäh ${ }^{1}$, Daniel Spies ${ }^{1}$, Rodrigo PeñaHernández $^{3}$, Raffaella Santoro ${ }^{3}$ and Constance Ciaudo ${ }^{1, *}$.

${ }^{1}$ Swiss Federal Institute of Technology Zurich, IMHS, Chair of RNAi and Genome Integrity, Zurich, Switzerland.

${ }^{2}$ Life Science Zurich Graduate School, University of Zürich, Switzerland.

${ }^{3}$ University of Zurich, Department of Molecular Mechanisms of Disease, Zurich, Switzerland

*Correspondence: cciaudo@ethz.ch

$£$ These authors contributed equally to this work

Running title: AGOs regulate a unique set of transcripts in mESCs.

Key words: Argonautes, KLF4, CTCF, H3K27me3, PRC2. 


\section{ABSTRACT}

The Argonaute proteins (AGO) are well-known for their essential role in posttranscriptional gene silencing in the microRNA (miRNA) biogenesis pathway. Only two AGOs (AGO1 and AGO2) are expressed in mouse embryonic stem cells (mESCs). The transcriptome of Ago mutant mESCs revealed a large and specific set of misregulated genes, compared to other miRNA biogenesis factor mutant cells, suggesting additional functions for the AGOs in stem cells. In this study, we endeavored to understand miRNA-independent roles of the AGOs in gene expression regulation through the integration of multiple datasets. Correlation of Ago mutant differential gene expression with ENCODE histone modification data of WT mESCs revealed that affected genes were regulated by the repressive histone modification H3K27me3. We validated this observation by performing chromatin immunoprecipitation followed by sequencing and observed a global loss of H3K27me3 in Ago mutant cells. Nevertheless, this reduction explains only a small part of the specific differential gene expression observed in Ago mutant mESCs. By integrating chromatin accessibility data in conjunction with prediction of transcription factor binding sites, we identified differential binding for five transcription factors, including KLF4 as a key modulator of more than half of the specific misregulation of gene expression in the absence of AGO proteins. Our findings illustrate that in addition to chromatin state, information about transcription factor binding is more revelatory in understanding the multi-layered mechanism adopted by cells to regulate gene expression. These data also highlight the importance of an integrative approach to unravel the variety of noncanonical functions of AGOs in mESCs. 


\section{INTRODUCTION}

The Argonaute (AGO) proteins are well known for their cytoplasmic role in the microRNA (miRNA) pathway, where they are key players involved in miRNAmediated translational inhibition of target mRNAs (Meister, 2013; Müller et al., 2020). However, more recently, several noncanonical functions, which are not directly linked to the cytoplasmic miRNA-mediated post-transcriptional gene silencing, have been described for the AGO proteins. For instance, several studies have reported nuclear functions for the AGO proteins, such as gene silencing and activation, alternative splicing, chromatin organization and, double-strand break repair (Li et al., 2020; Meister, 2013). Noticeably, most of these functions have been reported in human cancer cell lines and possible noncanonical functions in other systems, such as mouse early development, are only just starting to be understood. In fact, one study reported nuclear localized AGO2 in mouse embryonic stem cells (mESCs) and described a role for AGO2 in post-transcriptional gene silencing within the nucleus (Sarshad et al., 2018). We also recently demonstrated that nuclear AGO1 is linked to the proper distribution of heterochromatin at pericentromeres in mESCs. The depletion of AGO1 disturbed heterochromatin formation at pericentromeric repeats and led to an upregulation of transcripts from those specific regions (Müller et al., 2021).

In mESCs, only two out of the four AGO proteins (AGO1-4) are robustly expressed (Boroviak et al., 2018; Lykke-Andersen et al., 2008; Müller et al., 2020). The deletion of either one of them does not affect the viability of the cells nor their potential to differentiate into the three embryonic germ layers ( $N g o n d o$ et al., 2018). Interestingly, upon Ago2 depletion in mESCs, AGO1 levels increase and AGO1 become enriched with miRNAs normally loaded in AGO2, indicating a compensation of AGO2 loss by AGO1 and a redundancy of the two protein functions (Ngondo et al., 2018). However, Ago2 knockout (KO) mESCs cannot differentiate towards the extraembryonic endoderm, and this defect could not be rescued by overexpressing AGO1 (Ngondo et al., 2018). Thus, even if AGO1 and AGO2 have overlapping functions, especially regarding miRNA-mediated translational inhibition, there seems to be differences in their functional repertoire raising the possibility for additional specialized functions in early embryonic development. 
Here, we showed that the comparative analyses of gene expression profiles of mESC lines depleted of key regulators of the miRNA pathway (Dgcr8_KO, Drosha_KO, Dicer_KO and Ago2\&1_KO) revealed a larger number of specifically differentially expressed genes (DEGs) in Ago2\&1_KO mESCs that are associated with the positive regulation of RNA metabolic processes. We found that Ago2\&1_KO mESCs have a global loss of H3K27me3. However, this H3K27me3 reduction in Ago2\&1_KO mESCs did not significantly impact gene expression. Analyses of chromatin accessibility and chromatin immunoprecipitation followed by sequencing (ChIP-seq) data identified the transcription factors CTCF and KLF4 as major regulators of Ago2\&1_KO specific DEGs. In summary, our analyses suggested that loss of AGO1 and AGO2 induced changes in H3K27me3 occupancy and gene expression independently of miRNA-pathways due to a misregulation of the key stem cell pluripotency transcription factor, KLF4, revealing a novel axis of gene regulation in mESCs. 


\section{RESULTS}

\section{AG02\&1 regulate the expression of a class of genes in mESCs that do not depend on the miRNA pathway}

In order to assess the consequences of the loss of the AGO proteins on mESC gene expression, we integrated available RNA-sequencing (RNA-seq) data from (Schäfer et al., 2021) (Dataset EV1). Previous transcriptomics analyses in multiple miRNA_KO (Dgcr8_KO, Drosha_KO, Dicer_KO and Ago2\&1_KO) and WT mESCs identified many DEGs in all these mutants (Schäfer et al., 2021). Here, we intersected the Ago2\&1_KO DEGs with DEGs from other miRNA_KO mESCs and identified 1793 genes that were specifically misregulated in the AGO1 and AGO2 depleted cells and not altered in any other miRNA_KO lines (Fig 1A). Notably, the lack of misregulation in the other mutants ruled out the possibility of direct regulation by miRNAs for these DEGs and there was no overlap with previously reported miRNA targets in mESCs (Fig 1A) (Schäfer et al., 2021). Out of the Ago2\&1_KO specific DEGs, more than half (1045) were downregulated and 748 were upregulated (log2 fold-change $+/-0.5, P$ value $<0.05$, Fig 1B, Dataset EV1). Since a loss of miRNA-mediated repression leads to increased target levels, the high number of downregulated genes again argues against a miRNA-mediated regulation and implies that they might be attributed to AGO-specific functions. Importantly, the deficiency of both AGO1 and AGO2 in mESCs caused this distinct transcriptomic profile, as single Ago1_KO and Ago2_KO mESCs only had few DEGs, as previously reported (Fig EV1A and B, Dataset EV1) (Müller et al., 2021; Ngondo et al., 2018). Further, the overlap between the single Ago1_KO and Ago2_KO DEGs was rather small, which supports only a partial compensatory role for AGO1 and AGO2 functions (Fig EV1C) (Müller et al., 2021; Ngondo et al., 2018).

In order to understand which pathways are affected in mESCs upon combined loss of AGO1 and AGO2, we performed gene ontology (GO) analysis of Ago2\&1_KO specific DEGs and found that they were enriched in processes linked to nuclear processes, RNA metabolism, and positive transcriptional gene regulation (Fig 1C). Thus, the function of AGO proteins is not only related to post-transcriptional gene silencing, but also to functions that are probably independent of the miRNA pathway.

\section{Combined loss of AGO2\&1 in mESCs causes global loss of H3K27me3}


To determine how the combined depletion of Ago2\&1 affects gene expression in mESCs, we intersected Ago2\&1_KO RNA-seq and ENCODE histone chromatin immunoprecipitation followed by sequencing (ChIP-seq) from mESCs with the same genetic background (Fig 2A). Specifically, we analyzed the occupancy of histones containing repressive marks (H3K9me3 \& H3K27me3), enhancer marks (H3K4me1 \& H3K27ac) and active marks (H3K9ac, H3K36me3 \& H3K4me3) at Ago2\&1_KO specifically up- and downregulated genes. As control, we measured the occupancy of modified histones at predicted functional miRNA targets (Schäfer et al., 2021) and expressed genes in mESCs. We detected only minor differences in the occupancy of active histone marks between the Ago2\&1_KO specifically up- and downregulated genes and the expressed genes in mESCs (Fig 2A). In contrast, we found an enrichment of H3K4me1, H3K27ac, and H3K27me3 at Ago2\&1_KO specifically upregulated genes compared to downregulated genes and expressed genes in mESCs. Further, the differential H3K27me3 occupancy at Ago2\&1_KO specifically up- and downregulated genes seemed to be rather correlating with the loss of AGO proteins and not due to a miRNA-mediated regulation, as miRNA target genes (Schäfer et al., 2021) are mainly more enriched in active histone marks than H3K27me3 (Fig 2A). Noticeably, recent results have linked AGO2 with H3K27me3 by showing a reduction of H3K27me3 at certain target loci upon Ago2 depletion in mESCs (Kelly et al., 2019). To determine whether the combined loss of AGO2\&1 affects H3K27me3 levels and gene expression, we first measured and compared the global levels of several histone marks in Ago2\&1_KO mESCs and WT mESCs by Western Blotting (WB) (Fig 2B). We observed a drastic reduction of H3K27me3 signal in Ago2\&1_KO mESCs compared to WT mESCs whereas the levels of other modified histones such as H3K9me3, H3K27ac, and H3K4me3 were not particularly affected. These results indicated that the combined loss of AGO2\&1 globally decreased H3K27me3 levels. PRC2 is the complex involved in the deposition of H3K27me3 mark (Margueron and Reinberg, 2011). Interestingly, it was recently reported in cancer cells that miRNAs reinforce the repression of Polycomb Repressive Complex 2 (PRC2) transcriptional targets through independent and feedforward regulatory networks (Shivram et al., 2019). In addition, several members of the PRC2 complex have been shown to be directly regulated by miRNAs in Drosophila (Kennerdell et al., 2018), but not in mESCs deleted for Dicer, excluding a direct regulation by miRNAs (Graham et al., 2016). In order to assess the integrity of 
the PRC2 complex in Ago2\&1_KO mESCs and the potential contribution of miRNA regulation, we measured the expression of SUZ12 and EZH2, two core proteins of PRC2, in our series of miRNA_KO mESC lines. We observed that especially SUZ12 and, to a lesser extent, also EZH2 were specifically downregulated at the protein level (Fig EV2A), but not at the RNA level (Fig EV2B), in Ago2\&1_KO mESCs. In contrast, and consistent with previous results (Graham et al., 2016), we did not observe changes in SUZ12 and EZH2 at protein and mRNA level in the other miRNA_KO mESC lines. These results indicated that AGO1 and AGO2 globally regulate $\mathrm{H} 3 \mathrm{~K} 27 \mathrm{me} 3$ levels by affecting the protein levels of two key components of PRC2 complex using mechanisms that are independent of miRNA-mediated posttranscriptional pathway.

Due to the differential H3K27me3 occupancy at Ago2\&1_KO specifically up- versus downregulated genes (Fig 2A) and the drastic global reduction of H3K27me3 in Ago2\&1_KO mESCs (Fig 2B) we analyze whether differential H3K27me3 levels affect gene expression. First, we performed H3K27me3 ChIP-seq in WT and Ago2\&1_KO mESCs (Fig 2C). To validate our H3K27me3 ChIP-seq in WT mESCs, we compared it to available H3K27me3 ChIP-seq data from ENCODE (Davis et al., 2018; Dunham et al., 2012), observing a clear overlap between the two ChIP-seq data (Fig 2C and D). Consistent with the WB analysis, the ChIP-seq data confirmed a genome-wide loss of H3K27me3 in Ago2\&1_KO mESCs. In order to determine whether H3K27me3 loss correlates with changes in the expression of the Ago2\&1_KO DEGs, we clustered ChIP-seq levels at transcript regions using kmeans ( $k=4)$ clustering (Dataset EV2) (Ramírez et al., 2016). The first cluster (cluster_1) represents the genes most strongly enriched in H3K27me3 in WT and highly overlaps with known bivalent genes in mESCs (Asenjo et al., 2020) (Fig 2C, EV2C, Dataset EV2), while the second, third and fourth clusters represent transcripts with minor H3K27me3 levels.

We expected the loss of the repressive histone mark H3K27me3 to lead to an observable upregulation of associated genes. Indeed, genes from cluster_1 and cluster_2, which show the strongest H3K27me3 levels and therefore the strongest loss thereof, show a tendency to be upregulated (Fig $2 \mathrm{E}$ and F). In contrast, genes from cluster_3 and cluster_4, which are not marked with H3K27me3 in WT, show no enrichment for upregulation (Fig. 2E, EV2F). However, we found that only $14 \%$ of the upregulated genes and $11 \%$ of the downregulated genes belong to cluster_1 (Fig 
2E, EV2D and EV2E, Dataset EV5). These results suggest that the loss of H3K27me3 has a minor impact on the expression of Ago2\&1_KO specific DEGs. Thus, other pathways might be specifically affected in the absence of the AGO proteins to explain the specific DEGs.

\section{Loss of AGO2\&1 affects chromatin accessibility in mESCs}

To determine how AGO2\&1 affect gene expression, we measured chromatin accessibility by ATAC-seq (Buenrostro et al., 2013). We identified 3137 regions exhibiting significant differential accessibility (DA) in Ago2\&1_KO versus WT (2290 sites with increased accessibility, 847 regions with decreased accessibility) (Fig $3 A$, EV3A, Dataset EV3). In contrast, only minor differences in chromatin accessibility were observed in single AGO mutants, suggesting that only the combined loss of AGO2\&1 can affect chromatin structure of mESCs (Fig EV3B and C). These observations are in parallel with the changes observed at the transcriptomic level in these mutant cell lines, where Ago2\&1_KO, but not the single KO mutants, exhibited a strongly perturbed transcriptome (Fig EV1A and B). This suggests that the Ago2\&1_KO specific DEGs might at least be partially explained by changes in chromatin accessibility.

Next, we retrieved genes that were associated with statistically significant DA regions (Dataset EV3) at their promoter regions (transcription start site (TSS)distance $<1 \mathrm{kbp}$ ) and studied their differential expression (DE as indicated by log2 fold-change) in Ago2\&1_KO mESCs. We observed that the DE of genes with increased chromatin accessibility showed a strong enrichment for increased expression, while genes with decreased chromatin accessibility showed a tendency for decreased expression levels (Fig. EV3D). The difference between the DE distributions of the two groups showed statistical significance (t-test $p<1.8 e-5)$. Nevertheless, out of the 384 genes that showed increased chromatin accessibility, only 21 were Ago2\&1_KO specific DEGs and only 14 of them were upregulated (Fig EV3D and E, Dataset EV3 and EV5). Further, none of Ago2\&1_KO specific downregulated genes showed significant decrease in promoter accessibility (Fig EV3E and G). Thus, chromatin opening at gene promoter regions alone is not sufficient to explain the specific differential gene expression observed in Ago2\&1_KO mESCs. 


\section{Transcription factors explain an important part of the specific gene expression in Ago2\&1_KO mESCs}

While chromatin accessibility can influence transcription factor (TF) binding (Spitz and Furlong, 2012), TF binding has conversely been suggested to modulate chromatin accessibility in many cases (Baek et al., 2017), potentially affecting the expression of associated genes. To assess whether TFs might mediate differential chromatin accessibility, we analyzed the DA specifically at promoter (TSS-distance < $1 \mathrm{kbp}$ ) and enhancer regions (González-Ramírez et al., 2021). We observed an increased chromatin accessibility at these regulatory elements in Ago2\&1_KO compared to WT mESCs (Fig 3B and C), suggesting increased TF activity. Next, we integrated our chromatin accessibility data with motif-based TF binding site (TFBS) predictions using diffTF (Fig 3D, Dataset EV4) (Berest et al., 2019). Notably, five TFs (CTCF, KLF4, ERR2, REST and MYC) showed highly significant differential binding (Fig 3D, Dataset EV4), which might impact gene expression of their downstream targets in Ago2\&1_KO mESCs. To assess this impact, we further associated the motif-based TFBS predictions from diffTF with genes, based on promoter- (TSSdistance < 1kbp) and enhancer-proximity (González-Ramírez et al., 2021), and compared DA at TFBS with differential gene expression (Fig 3E and F, Datasets EV4 and EV5). For most TFs, a notable positive correlation between DA and DE was observable, indicating that differential binding of TFs indeed seemed to affect gene expression, thus potentially explaining Ago2\&1_KO specific DEGs. Combined, the five identified TFs positively correlate with 289 Ago2\&1_KO specific DEGs (152 upand 137 downregulated), from which CTCF and KLF4 contributed the most (149 and 147 genes respectively, Fig 3E and F, Dataset EV4 and EV5).

In summary, we observed an increase in chromatin accessibility at promoters and enhancers in Ago2\&1_KO mESCs and identified five TFs showing highly significant DA at their predicted binding sites. Association of DA at TFBS with proximal genes correlated with around 17\% of the Ago2\&1_KO specific DEGs of which KLF4 and CTCF binding sites correspond to the largest portion (>13\%).

\section{KLF4 regulates the majority of Ago2\&1_KO specific DEGs}

Motivated by the relatively large number of genes predicted as KLF4 and CTCF targets, we further investigated these two TFs. CTCF is well-known for its role in chromatin looping and organization (Oudelaar and Higgs, 2021). More recently, 
KLF4 has also been linked to chromatin organization during the reprogramming of mouse embryonic fibroblasts (MEFs) to induced pluripotent stem cells (iPSCs) by conferring enhancer-promoter contacts (Di Giammartino et al., 2019). Thus, altering CTCF and KLF4 levels or their binding site accessibility might affect the interaction of regulatory elements and the underlying gene expression. We did not observe a change in Ctcf expression at RNA nor protein levels by qPCR and by WB in Ago2\&1_KO mESCs (Fig EV4A and B). In contrast, KLF4 was strongly downregulated in Ago2\&1_KO compared to WT mESCs (Fig 4A, Dataset EV1), suggesting that the decrease in KLF4 levels might affect gene expression in Ago2\&1_KO mESCs. Despite the strong differences in the observed misregulation between the two TFs, they had been associated with a similar number of target genes by our TFBS motif and ATAC-seq-based analysis. ChIP-seq has been recently performed in mESCs for both TFs allowing us to assess and study their targets with higher accuracy (Di Giammartino et al., 2019; Nora et al., 2017).

Consistent with the increase in chromatin accessibility at CTCF TFBS in Ago2\&1_KO cells (Fig 3D), we observed that the number of ChIP-seq-indicated CTCF-bound promoters and enhancers was higher for the upregulated Ago2\&1_KO specific genes $(35 \%, 260)$ than for the downregulated ones $(19 \%, 201)$ (Fig $4 \mathrm{~B}$, Dataset EV5). While the ChIP-seq-based analysis for CTCF substantially increased the number of explained genes from $8 \%$ to $25 \%$ (compared to the motif-/ATAC-seqbased approach), interestingly, we found that a much larger fraction of the promoter and enhancer regions of Ago2\&1_KO specific DEGs were bound by KLF4 (upregulated genes, 497, 73\%; downregulated genes, 47\%, 550) suggesting a major role in the regulation of Ago2\&1_KO specific DEGs (Fig 4C, Dataset EV5).

In conclusion, the analysis of different genomics datasets allowed us to identify KLF4 as the major contributor to the Ago2\&1_KO DEGs (Fig. 4D and E, Dataset EV5).

\section{DISCUSSION}

In this work, we showed that AGO2\&1 regulate specific gene expression in mESCs. The transcriptome analyses of Ago2\&1_KO mESCs and other miRNA mutant mESCs indicated that the change in gene expression upon loss of AGO2\&1 can be attributed only to a small degree by the loss of miRNA-mediated regulation, underlying a non-canonical function of AGO2\&1 in mESCs. Here, we have studied 
potential mechanisms of how AGO2\&1 can affect gene expression and identified KLF4 as the major mediator of AGO2\&1 regulated genes.

Given that the miRNA effector proteins (DGCR8, DROSHA, DICER and AGO2\&1), are involved in the canonical miRNA biogenesis pathway, one might assume that their knockouts would lead to similar transcriptomic perturbations. As we observed previously (Schäfer et al., 2021), DEGs in Ago2\&1_KO showed strong similarities to Dicer_KO, but not to Dgcr8_KO or Drosha_KO. This might be partially attributable to noncanonical miRNA pathways, which function independently of the Microprocessor (DGCR8/DROSHA), but still require DICER and the AGO proteins (Bodak et al., 2017a). Thus, a limited set of miRNAs might still be active in Dgcr8_KO, Drosha_KO but not in Dicer_KO and Ago2\&1_KO. Furthermore, AGO2 protein levels are strongly reduced in miRNA_KO mESCs due to its targeted proteasome degradation in the absence of miRNAs (Bodak et al., 2017b; Smibert et al., 2013). Some miRNAindependent AGO-mediated functions might therefore also be partially affected in miRNA_KO mESC lines. Surprisingly, despite these similarities, comparing DEGs between Ago2\&1_KO and other miRNA_KO mESCs revealed a large number of DEGs that were specific to individual mutants and especially to Ago2\&1_KO (Fig $1 A)$, indicating potential miRNA-independent AGO specific functions. Indeed AGO specific functions, for example nuclear functions, have already been described in cancer cell lines and more recently also in mESCs (Li et al., 2020; Meister, 2013; Müller et al., 2021; Sarshad et al., 2018). Interestingly, we recently reported a global redistribution of H3K9me3 upon Ago1 depletion in mESCs, however, this had very little impact on gene expression (Fig EV1A) (Müller et al., 2021). Similarly, Ago2_KO transcriptome also showed largely unchanged gene expression levels (Fig EV1A) (Ngondo et al., 2018). Given the strong observed perturbation in the Ago2\&1_KO transcriptome, this might indicate that $A G O 1$ and $A G O 2$ do have global compensatory mechanisms in mESCs such that only disrupting the function of both proteins impacts gene expression strongly.

One such function is the regulation of SUZ12 and EZH2, two key components of PRC2. We showed that SUZ12 and EZH2 in Ago2\&1_KO mESCs are reduced at protein levels but not at mRNA levels. As consequence, the amount of H3K27me3 in cells lacking AGO2\&1 is globally reduced. However, the loss of H3K27me3 is not sufficient to fully explain the gene expression changes observed in Ago2\&1_KO mESCs. 
The integrative analyses of differential chromatin accessibility at predicted TFBS revealed a strong regulatory potential of two TFs, CTCF and, in particularly, KLF4, that can display both an activating and a repressive function (Bialkowska et al., 2017). Indeed, we found that KLF4 binds the promoter of about $50-70 \%$ of Ago2\&1_KO specific up- and downregulated genes, respectively. Importantly, we also observed that the expression of KLF4 is strongly reduced in Ago2\&1_KO mESCs. KLF4 is a pluripotency factor, which has been reported to occupy promoters of other key pluripotent transcription factors OCT4/SOX2/NANOG (OSN), thereby affecting their expression and vice versa (Bialkowska et al., 2017; Jiang et al., 2008). In addition, high KLF4 levels are associated with pluripotency and low levels with the onset of differentiation (Bialkowska et al., 2017). Accordingly, recent RNA-seq and Ribo-seq data (Schäfer et al., 2021) indicated that the pluripotency factors Nanog and Oct4 are upregulated in Ago2\&1_KO mESCs. Further, it has also been reported that in the absence of KLF4, the expression of two other Krüppel-life factors (KLF2, KLF5) increase, probably in order to compensate for KLF4 loss (Bialkowska et al., 2017; Di Giammartino et al., 2019). Indeed, we also observed an increase in the expression of KIf2, but not KIf5, in Ago2\&1_KO mESCs (Dataset EV1). However, functional studies would be needed to confirm whether this increase potentially compensates for some of the loss of KLF4 in Ago2\&1_KO. Thus, it might be interesting in the future to assess in detail the pluripotency status of these cells.

Interestingly, KLF4 has also been linked to other functions apart from regulating the pluripotency network. Recently, KLF4 has been shown to interact with SUZ12, a PRC2 complex member in mESCs (Di Giammartino et al., 2019). The observed reduction of SUZ12 at protein levels in Ago2\&1_KO mESCs (Fig EV2A) suggests that the loss of KLF4 might destabilize SUZ12. This might be worth further investigations in the future, also with regard to the deposition of H3K27me3 in Ago2\&1_KO mESCs, which then might be attributed to a secondary impact due to the loss of KLF4 in these mutant cells.

KLF4 has also been implicated in genome reorganization in mESCs and important functions in conferring enhancer connectivity (Di Giammartino et al., 2019). There, the disruption of KLF4 and its binding sites in mESCs showed an abrogation of enhancer contacts, which consequently decreased expression levels of associated genes (Di Giammartino et al., 2019). The loss of KLF4, along with many AGO2\&1regulated genes in our study, potentially may lead to alterations in chromatin 
conformation, which might be linked directly or indirectly to the AGO proteins. Indeed, previous studies already pointed towards potential alterations of the chromatin conformation linked to the AGO proteins (Moshkovich et al., 2011; Shuaib et al., 2019). In Drosophila, AGO2 has already been shown to interact with the architectural protein CTCF and to localize to chromatin regions, while abrogating this interaction led to reduced chromatin looping (Moshkovich et al., 2011). Also, in human cells, AGO1 was reported to be required for 3D chromatin maintenance. One study described that the loss of AGO1 leads to a disorganization of chromatin structure, which subsequently perturbed gene expression (Shuaib et al., 2019). Taken together, our observations as well as previous studies suggest a potential link for the AGO proteins in the modulation of chromatin conformation. Thus, mESCs might provide a unique opportunity to further investigate chromatin-related functions of the AGOs in mammals.

In conclusion, our study revealed non-canonical functions of AGO2\&1 in mESCs that do not overlap with the canonical miRNA pathways and revealed a novel axis of gene regulation through the transcription factor KLF4.

\section{MATERIALS AND METHODS}

\section{Mouse ESC lines}

WT and all mutant E14 mESC lines (129/Ola background) were cultured as described in (Müller et al., 2021; Ngondo et al., 2018; Schäfer et al., 2021).

\section{CRISPR/Cas9 mediated gene knockout}

The generation of the Dgcr8_KO mESCs and Drosha_KO mESCs cell lines were previously published by (Cirera-Salinas et al., 2017). The Dicer_KO mESCs lines were previously published by (Bodak et al., 2017b). The Ago2\&1_KO mESCs were previously published by (Schäfer et al., 2021).

\section{Protein extraction and Western Blot Analysis}

Protein extraction was performed in RIPA lysis buffer (50 mM Tris-HCL pH 8.0, $150 \mathrm{mM} \mathrm{NaCl}, 1 \%$ IGEPAL CA-630, 0.5\% sodium deoxycholate, $0.5 \%$ sodium dodecyl sulfate supplemented with EDTA- 
free protease inhibitor cocktail (Roche)). The cell pellet was resuspended in ice-cold RIPA buffer and sonicated twice for 10 seconds. Afterwards samples were centrifuged $10 \mathrm{~min}$ at $10000 \mathrm{rpm}$ and the supernatant was retrieved in a new $1.5 \mathrm{ml}$ Eppendorf tube. The concentration of the protein extraction was assessed with a Bradford assay (Bio-Rad Laboratories). Laemmli buffer to a concentration of $1 \mathrm{x}$ was added to the samples and the samples were denatured for 5 min at $95^{\circ} \mathrm{C}$. Western blotting has been performed as described in (Müller et al., 2021). Antibodies used for the WBs were: TUBULIN (Sigma-Aldrich T6199, 1:10000), H3K27me3 (ab6002, 1:2000), H3K27ac (ab177178, 1:2000), H3K4me3 (ab8580, 1:2000), H3K9me3 (ab8898, 1:2000), H3 (ab1791, 1:2000), SUZ12 (CST \#3737), EZH2 (CST \#5246), CTCF (ActiveMotif 61311, 1:2000), KLF4 (R\&D AF3158, 1:2000).

\section{RNA extraction and quantitative RT PCR Analysis}

The RNA extraction has been carried out as described in (Bodak and Ciaudo, 2016). Briefly, RNA extraction from cell pellets was performed with the Trizol reagent (Life Technologies). $1 \mathrm{ml}$ Trizol was added to the cell pellet and mixed by vortexing for 10 seconds. Then, $200 \mu$ l Chloroform (SigmaAldrich) was added, and samples were vortexed again for 10 seconds. Samples were centrifuged at $4^{\circ} \mathrm{C}$ for $15 \mathrm{~min}$ at $13500 \mathrm{xg}$ and the upper phase was transferred into a new $1.5 \mathrm{ml}$ Eppendorf tube. $600 \mu \mathrm{l}$ isopropanol (Merck) was added, mixed by vortexing and samples were centrifuged for $30 \mathrm{~min}$ at $4^{\circ} \mathrm{C}$ at $13500 \mathrm{xg}$. The supernatant was removed, and the RNA pellet was washed once with $1 \mathrm{ml}$ ice-cold $70 \%$ Ethanol and centrifuged again for $10 \mathrm{~min}$ at $13500 \mathrm{xg}$. The supernatant was removed, the RNA pellet was air-dried and resuspended in RNase-free water. The quality of $1 \mu \mathrm{g}$ of RNA was checked by on a $1 \%$ agarose gel (Sigma). RT and qPCR has been performed as described by (Müller et al., 2021). Primers are listed in Table EV6.

\section{Reference genome and gene annotation}

All OMICs analyses were based on the mouse reference genome GRCm38/mm10. ChIP-seq analyses were performed using GENCODE mouse gene annotations in version 23 (Frankish et al., 2019) and Drosophila genome in version 6 (Hoskins et al., 2015) for spike-in normalization, while all other analyses were performed using the comprehensive mouse gene annotation file from ENSEMBL in version 98 (Cunningham et al., 2019). 


\section{RNA-seq analysis of RNAi mutants}

RNA-seq data was obtained from GEO (Table EV6) and analyzed as described in (Schäfer et al., 2021). Briefly, the snakePipes RNA-seq pipeline (Bhardwaj et al., 2019) was employed with default arguments for trimming, quality control, mapping, read counting and differential expression analysis using Trim Galore/cutadapt (Martin, 2011), FastQC/multiQC (Andrews et al., 2012), Bowtie2 (Langmead and Salzberg, 2012), featureCounts (Liao et al., 2014) and DESeq2 (Love et al., 2014) respectively.

\section{Definition of Ago2\&1_KO-specific DEGs}

DEGs in each of the miRNA_KO mutants were defined with an adjusted p-value threshold of 0.05 and a minimal log2FoldChange of 0.5 . The set of Ago2\&1_KO-specific misregulated genes described throughout this paper (Dataset EV1) consists of genes that are differentially expressed in Ago2\&1_KO and not differentially expressed in any of the other miRNA_KO mutants (Dgcr8_KO, Drosha_KO, Dicer_KO). Visualizations of differential expression and gene set overlaps were realized as MA-plots and Venn-diagrams using matplotlib/seaborn (Hunter, 2007; Waskom, 2021) and eulerr/matplotlib_venn (Larsson et al., 2018, http://eulerr.co/) respectively.

For the gene ontology analysis, the ClueGO App (Bindea et al., 2009) for Cytoscape (Shannon et al., 2003) was used. For the analysis, the terms 'Biological Process', 'Molecular Function' and 'Cellular Component' were used (date: 2020/03/09). Only pathways with pValues $<0.005$ were considered and Terms/Pathways with a minimum GO tree interval level of 3 and a maximum of 10 were used. To represent the size of the nodes according to their pValues, the Bonferroni step down corrected Term PValue was selected and the - $\log 10$ of these pValues was calculated and represented in the figure.

\section{Analysis of histone mark levels for gene groups}

Gene groups were defined as follows. Ago2\&1_KO specific DEGs were split based on their differential expression into an upregulated and a downregulated group. Predicted MiRNA Targets were taken as provided by (Schäfer et al., 2021). Expressed genes were defined as genes from the comprehensive gene annotation set from ENSEMBL (described above) with a minimum expression of 
1 TPM in our WT RNA-seq data (Dataset EV1). For each gene, all annotated transcripts were considered as separated instances. Each transcript was converted to a range of $10 \mathrm{kbp}$ around the transcription start site in order to account for the diverse placement patterns of different histone marks.

Genome-wide histone mark levels for seven different marks were obtained from ENCODE experiment IDs ENCSR000CGO, ENCSR000CGN, ENCSR000CGQ, ENCSR000CGP, ENCSR000CGR, ENCSR000ADM, ENCSR059MBO, Davis et al., 2018; Dunham et al., 2012) as bigwig files of signal over input log2FoldChanges for two combined replicates. For each region in each of the described groups, the mean signal was obtained for each histone mark using the multiBigwigSummary tool (Ramírez et al., 2016). Finally, the mean signals were averaged across all transcripts of each gene group and z-score normalization was applied on a per histone mark basis.

\section{H3K27me3 ChIP-seq}

\section{Sample and library preparation}

The chromatin extraction and pull-down has been performed as described in (Müller et al., 2021). In order to prepare chromatin for sequencing, two replicates have been performed, where $100 \mu \mathrm{g}$ of chromatin, together $125 \mathrm{ng}$ Drosophila spike-in chromatin (a kind gift from the Santoro lab), was precleared with $50 \mu \mathrm{l}$ of Dynabeads protein G (ThermoFisher Scientific) and further used for the chromatin pull-down. $10 \mu \mathrm{g}$ of H3K27me3 (ab6002) was used for the pull-down.

Library preparation and sequencing was performed by the Functional Genomics Center Zürich (FGCZ). For the library preparation the NEBNext ${ }^{\circledR}$ Ultra ${ }^{\text {TM }}$ II DNA Library Prep Kit from NEB (New England Biolabs, Ipswich, MA) was used. $1 \mathrm{ng}$ starting material of each sample was used, endrepaired and afterwards adenylated. Then, indexed adapters were ligated to the fragmented samples and PCR was performed to enrich the fragments. The library quality and quantity were assessed by with the help of a Qubit $\AA^{\circledR}$ (1.0) Fluorometer and a Tapestation (Agilent, Waldbronn, Germany). The libraries were sequenced on the Illumina Novaseq 6000 (Illumina, Inc, California, USA) by 100 bp single reads.

\section{ChIP-seq analysis pipeline}


Adaptors were trimmed using trimmomatic (Bolger et al., 2014). Sequencing reads were mapped to the mouse mm10 genome and to the Drosophila dm6 genome using bowtie2 (Langmead and Salzberg, 2012). Duplicates were marked and filtered out using samtools (Li et al., 2009). bamCompare from deepTools (Ramírez et al., 2016) was used to generate a bigwig file of IP over Input. Drosophila-mapped read counts were used as scaling factor for the Input and the IP samples in order to get normalized read counts.

\section{Cluster-analysis of transcript-centric histone modification levels}

Both WT ChIP-seq replicates were compared to the WT H3K27me3 ChIP-seq data from ENCODE (ENCSR059MBO, Davis et al., 2018; Dunham et al., 2012). Compared to the already published ENCODE ChIP-seq, one replicate exhibited stronger signal-to-noise ratio and this sample and the according Ago2\&1_KO replicate were selected for representative images and cluster analysis in Fig. 2 and EV2.

The published H3K27me3 ChIP-seq data from ENCODE (ENCSR059MBO, as described above) was compared to our generated data for experimental validation. To improve comparability and account for differences in samples preparation and data analysis between the published and our data, signal levels of the ENCODE data were normalized to our WT sample (scaling with a factor of $1 / 3$ ). Clustered heatmaps with mean profiles for four clusters were generated using deeptools' computeMatrix and plotHeatmap tools for the complete set of annotated transcripts around TSS $(<5$ kbp distance). Transcripts from each cluster were reduced to their corresponding genes and associated with differential expression in Ago2\&1_KO for comparison of log2FoldChange distributions across the four clusters using CDF curves plotted with matplotlib/seaborn (Hunter, 2007; Waskom, 2021).

For the comparison of the H3K27me3 ChIP-seq cluster_1 with known bivalent genes, the list of highconfidence bivalent "HC-Bivalent" genes from (Asenjo et al., 2020) was retrieved.

The pyGenomeTracks tool was used in order to show a genome browser view of selected regions (Ramírez et al., 2018).

\section{ATAC-seq}


bioRxiv preprint doi: https:/doi.org/10.1101/2021.10.18.464771; this version posted October 19,2021 . The copyright holder for this preprint (which was not certified by peer review) is the author/funder, who has granted bioRxiv a license to display the preprint in perpetuity. It is made available under aCC-BY-NC-ND 4.0 International license.

Two millions of cells were trypsinized and resuspended in $1 \mathrm{ml}$ of freezing medium (serum $+10 \%$ DMSO). Cells were transferred into a freezing container and stored at $-80^{\circ} \mathrm{C}$. ATAC-seq sample preparation, library preparation and sequencing have been performed by Quick Biology (https://www.quickbiology.com/ngs-services/ATAC-seq-service) according to (Corces et al., 2017) with 50,000 intact cells. Cells were first washed, then lysed to obtain nuclei preparation. Using aTn5 transposase, the genome was simultaneously fragmented and tagmented, leading to amplifiable DNA fragments with sequencing adapters for the Illumina platform. The fragments were amplified by PCR and purified using Qiagen MinElute PCR Purification Kit (Qiagen, Maryland, USA). The final library quality was controlled using the Agilent Bioanalyzer 2100 (Agilent Technologies, Santa Clara, CA) and the quantity assessed using the Life Technologies Qubit 3.0 Fluorometer (Life Technologies, Carlsbad, CA). Finally, the libraries were sequenced on an Illumina HiSeq X Ten Sequencer (Illumnia Inc., San Diego, CA) with 2 x 150 bp read (paired end).

\section{ATAC-seq analysis pipeline}

Raw read counts were processed by the snakePipes DNA-mapping and ATAC-seq pipeline (Bhardwaj et al., 2019). Briefly, reads were trimmed using Trim Galore/cutadapt (Martin, 2011) with parameters "-nextera -paired" after which Bowtie2 was called for mapping with default arguments. Reads were filtered for PCR duplicates and mappings were filtered if mapping quality was below "3" or if the fragment size was smaller than $150 \mathrm{bp}$, to only keep reads originating from nucleosome free regions (NFRs). Genrich (available at https://github.com/jsh58/Genrich) was used for peak calling and CSAW/EdgeR (Lun and Smyth, 2016) determined Differential Accessibility (DA) of the called peak regions. Analysis of quality control metrics indicated differences in experimental ATAC efficiencies across samples. Therefore, fraction of reads in peaks (FRiPs) score was used as linear scaling factors in downstream analyses (differential accessibility, MA plots, heatmap/profile plots, diffTF analysis (see below)) to compensate for differences across samples, as suggested by (Reske et al., 2020).

Comparison of WT and Ago2\&1_KO were performed using (i) deeptools heatmap/profile plots over gene regions, similarly as described in the H3K27me3 ChIP-seq analysis, (ii) profile plots at promoter regions ( $<3 \mathrm{kbp}$ TSS distance of all annotated transcripts) using deeptools computeMatrix and plotProfile (iii) profile plots at enhancer regions, as determined by (González-Ramírez et al., 2021) (iv) 
MA plots of the differential accessibility at peak regions (using matplotlib/seaborn, Hunter, 2007; Waskom, 2021).

\section{Integration with RNA-seq and TF motifs}

Peaks with statisticaly significant DA $(p<0.05)$ were associated with genes that were in $1000 \mathrm{bp}$ proximity to their transcription start sites. Genes associated with increased chromatin accessibility and decreased accessibility were grouped separately and associated with differential expression as assessed by RNA-seq, which allowed for a comparison of their log2FoldChange distributions (CDF plots were generated using matplotlib/seaborn, Hunter, 2007; Waskom, 2021),

The diffTF tool (Berest et al., 2019) was employed to assess potential differential binding of Transcription Factors (TFs), based on chromatin accessibility data. The tool relies on previously determined binding motifs for a large set of transcription factors and assesses DA at predicted motifbased TF binding sites (TFBS) within the genome. Per-TFBS information and per-TF summary data is generated and indicates whether TF binding sites were associated with increased or decreased DA. diffTF was run on all TFs having minimal expression of 1 TPM in our WT RNA-seq data and with a binding motif available as obtained from the HOCOMOCO database (v10, Kulakovskiy et al., 2018).

TFBS-level information was obtained for the 5 TFs with most significant DA at TFBSs, filtered for statistical significant DA $(p<0.05)$ and associated with genes in two ways: (i) promoter-based association was performed using bedtools closest (Quinlan and Hall, 2010) on gene TSSs with a 1000 bp distance filter, (ii) enhancer-based association was performed based on enhancer-gene associations as provided by (González-Ramírez et al., 2021, "Hi-C-top" active and poised enhancers were combined); TFBSs that were overlapping with reported enhancer regions were associated with the corresponding genes. Associated DA and DEG information was then visualized on a per-gene basis using scatterplots (Hunter, 2007; Waskom, 2021).

\section{Integration of published KLF4- and CTCF-ChIP-seq data}

CTCF ChIP-seq peaks were obtained from (Nora et al., 2017). KLF4 ChIP-seq peaks were obtained from (Di Giammartino et al., 2019), where all reported peaks but "Transient"-labeled ones were considered. 
Peaks were associated with genes again by promoter proximity $(<1000$ bp to TSS) and enhancer overlap (González-Ramírez et al., 2021) similarly to as indicated in the last section, but using pybedtools (Dale et al., 2011; Quinlan and Hall, 2010). Associated genes were filtered for DEGs in Ago2\&1_KO and used for Venn diagram overlaps with previously defined gene groups.

\section{Combined contribution of regulatory mechanisms to Ago2\&1_KO-specific DEGs}

Gene sets derived from the previously described mechanisms (H3K27me3, chromatin accessibility, general TF-binding, CTCF- and KLF4-binding) were intersected with the set of Ago2\&1_KO-specific DEGs and overlapped using an UpSet plot in a union-fashion, as implemented by (Gu et al., 2016).

\section{QUANTIFICATION AND STATISTICAL ANALYSIS}

See Methods Details on how quantification and statistical analyses have been performed. If not mentioned otherwise, statistical analysis has been performed using PRIMS 8 more detail is indicated in the figure legends.

\section{DATA AVAILABILITY}

Data visualization has been performed with the tools, described in the Method sections (Table EV6). If not mentioned otherwise graphs have been generated by using PRIMS.

All code for data analyses and visualizations described in the paper are found in the following github repository https://github.com/moritzschaefer/ago21_specific_effects.

Sequencing data has been deposited on GEO (Table EV6)

The ATAC-seq and ChIP-seq data has been deposited to the GEO database: GSE185410 Reviewer access token: gnszaiwsbrunpwt

\section{Acknowledgments}

We would like to thank the members of the Ciaudo lab and Dr. Tobias Beyer for fruitful discussions and the critical reading of this manuscript. This work was supported by the Swiss National Science Foundation (grants 31003A_173120 and 310030_196861) to C.C. In 
addition, C.C, M.M and R.S were supported by the NCCR RNA and Disease. We also want to thank the Functional Genomics Center Zurich (FGCZ) for their support with the preparation of ChiP-seq libraries and sequencing. We also want to thank Quick Biology for their help with the ATAC-seq library preparation, sequencing and computational support.

\title{
Author Contributions
}

Conceptualization, MM, MS and CC; laboratory experiments, MM, TF; ChiP-seq libraries preparation, MM, RPH; computational analysis, MS and DS; writing original draft preparation, MS, MM and CC; writing, review and editing, CC; expertise and editing, RS; visualization, MS, MM and CC.; supervision, CC; funding acquisition, CC. All authors have read and agreed to the published version of the manuscript.

\section{Conflict of Interests}

The authors declare no financial and non-financial competing interests.

\section{Supporting Information}

\author{
Expanded View Figures \\ Dataset EV1 \\ Dataset EV2 \\ Dataset EV3 \\ Dataset EV4 \\ Dataset EV5 \\ Table EV6
}

\section{References}

Andrews, S., Krueger, F., Segonds-Pichon, A., Biggins, L., Krueger, C., and Wingett, S. (2012). FastQC.

Asenjo, H.G., Gallardo, A., López-Onieva, L., Tejada, I., Martorell-Marugán, J., Carmona-Sáez, P., and Landeira, D. (2020). Polycomb regulation is coupled to cell cycle transition in pluripotent stem cells. Sci. Adv. 6.

Baek, S., Goldstein, I., and Hager, G.L. (2017). Bivariate Genomic Footprinting Detects Changes in Transcription Factor Activity. Cell Rep. 19, 1710-1722.

Bannister, A.J., and Kouzarides, T. (2011). Regulation of chromatin by histone modifications. Cell Res. 21, 381-395. 
Berest, I., Arnold, C., Reyes-Palomares, A., Palla, G., Rasmussen, K.D., Giles, H., Bruch, P.M., Huber, W., Dietrich, S., Helin, K., et al. (2019). Quantification of Differential Transcription Factor Activity and Multiomics-Based Classification into Activators and Repressors: diffTF. Cell Rep. 29, 3147-3159.e12.

Bhardwaj, V., Heyne, S., Sikora, K., Rabbani, L., Rauer, M., Kilpert, F., Richter, A.S., Ryan, D.P., and Manke, T. (2019). SnakePipes: Facilitating flexible, scalable and integrative epigenomic analysis. Bioinformatics 35, 4757-4759.

Bialkowska, A.B., Yang, V.W., and Mallipattu, S.K. (2017). Krüppel-like factors in mammalian stem cells and development. Dev. 144, 737-754.

Bindea, G., Mlecnik, B., Hackl, H., Charoentong, P., Tosolini, M., Kirilovsky, A., Fridman, W.H., Pagès, F., Trajanoski, Z., and Galon, J. (2009). ClueGO: A Cytoscape plug-in to decipher functionally grouped gene ontology and pathway annotation networks. Bioinformatics 25, 1091-1093.

Bodak, M., and Ciaudo, C. (2016). Monitoring Long Interspersed Nuclear Element 1 Expression During Mouse Embryonic Stem Cell Differentiation. Methods Mol. Biol. 1400, 237-259.

Bodak, M., Cirera-Salinas, D., Luitz, J., and Ciaudo, C. (2017a). The Role of RNA Interference in Stem Cell Biology: Beyond the Mutant Phenotypes. J. Mol. Biol. 429, 1532-1543.

Bodak, M., Cirera-Salinas, D., Yu, J., Ngondo, R.P., and Ciaudo, C. (2017b). Dicer, a new regulator of pluripotency exit and LINE-1 elements in mouse embryonic stem cells. FEBS Open Bio 7, 204-220.

Bolger, A.M., Lohse, M., and Usadel, B. (2014). Trimmomatic: A flexible trimmer for Illumina sequence data. Bioinformatics 30, 2114-2120.

Boroviak, T., Stirparo, G.G., Dietmann, S., Hernando-Herraez, I., Mohammed, H., Reik, W., Smith, A., Sasaki, E., Nichols, J., and Bertone, P. (2018). Single cell transcriptome analysis of human, marmoset and mouse embryos reveals common and divergent features of preimplantation development. Development 145, dev167833.

Buenrostro, J.D., Giresi, P.G., Zaba, L.C., Chang, H.Y., and Greenleaf, W.J. (2013). Transposition of native chromatin for fast and sensitive epigenomic profiling of open chromatin, DNA-binding proteins and nucleosome position. Nat. Methods 10,1213-1218.

Cirera-Salinas, D., Yu, J., Bodak, M., Ngondo, R.P., Herbert, K.M., and Ciaudo, C. (2017). Noncanonical function of DGCR8 controls mESC exit from pluripotency. J. Cell Biol. 216, 355-366.

Corces, M.R., Trevino, A.E., Hamilton, E.G., Greenside, P.G., Sinnott-Armstrong, N.A., Vesuna, S., Satpathy, A.T., Rubin, A.J., Montine, K.S., Wu, B., et al. (2017). An improved ATAC-seq protocol reduces background and enables interrogation of frozen tissues. Nat. Methods 14, 959-962.

Cunningham, F., Achuthan, P., Akanni, W., Allen, J., Amode, M.R., Armean, I.M., Bennett, R., Bhai, J., Billis, K., Boddu, S., et al. (2019). Ensembl 2019. Nucleic Acids Res. 47, D745-D751.

Dale, R.K., Pedersen, B.S., and Quinlan, A.R. (2011). Pybedtools: A flexible Python library for manipulating genomic datasets and annotations. Bioinformatics 27, 3423-3424.

Davis, C.A., Hitz, B.C., Sloan, C.A., Chan, E.T., Davidson, J.M., Gabdank, I., Hilton, J.A., Jain, K., Baymuradov, U.K., Narayanan, A.K., et al. (2018). The Encyclopedia of DNA elements (ENCODE): Data portal update. Nucleic Acids Res. 46, D794-D801.

Dobin, A., Davis, C.A., Schlesinger, F., Drenkow, J., Zaleski, C., Jha, S., Batut, P., Chaisson, M., and Gingeras, T.R. (2013). STAR: Ultrafast universal RNA-seq aligner. Bioinformatics 29, 15-21.

Dunham, I., Kundaje, A., Aldred, S.F., Collins, P.J., Davis, C.A., Doyle, F., Epstein, C.B., Frietze, S., Harrow, J., Kaul, R., et al. (2012). An integrated encyclopedia of DNA elements in the human genome. Nature 489, 57-74.

Frankish, A., Diekhans, M., Ferreira, A.-M., Johnson, R., Jungreis, I., Loveland, J., Mudge, J.M., Sisu, 
C., Wright, J., Armstrong, J., et al. (2019). GENCODE reference annotation for the human and mouse genomes. Nucleic Acids Res. 47, D766-D773.

Di Giammartino, D.C., Kloetgen, A., Polyzos, A., Liu, Y., Kim, D., Murphy, D., Abuhashem, A., Cavaliere, P., Aronson, B., Shah, V., et al. (2019). KLF4 is involved in the organization and regulation of pluripotency-associated three-dimensional enhancer networks. Nat. Cell Biol. 21, 1179-1190.

González-Ramírez, M., Ballaré, C., Mugianesi, F., Beringer, M., Santanach, A., Blanco, E., and Di Croce, L. (2021). Differential contribution to gene expression prediction of histone modifications at enhancers or promoters. PLOS Comput. Biol. 17, e1009368.

Graham, B., Marcais, A., Dharmalingam, G., Carroll, T., Kanellopoulou, C., Graumann, J., Nesterova, T.B., Bermange, A., Brazauskas, P., Xella, B., et al. (2016). MicroRNAs of the miR-290-295 Family Maintain Bivalency in Mouse Embryonic Stem Cells. Stem Cell Reports 6, 635-642.

Gu, Z., Eils, R., and Schlesner, M. (2016). Complex heatmaps reveal patterns and correlations in multidimensional genomic data. Bioinformatics 32, 2847-2849.

Harris, C.R., Millman, K.J., van der Walt, S.J., Gommers, R., Virtanen, P., Cournapeau, D., Wieser, E., Taylor, J., Berg, S., Smith, N.J., et al. (2020). Array programming with NumPy. Nature 585, 357362.

Hoskins, R.A., Carlson, J.W., Wan, K.H., Park, S., Mendez, I., Galle, S.E., Booth, B.W., Pfeiffer, B.D., George, R.A., Svirskas, R., et al. (2015). The Release 6 reference sequence of the Drosophilamelanogaster genome. Genome Res. 25, 445.

Hunter, J.D. (2007). MATPLOTLIB: A 2D GRAPHICS ENVIRONMENT. Comput. Sci. Eng. 9, 90-95.

Jiang, J., Chan, Y.S., Loh, Y.H., Cai, J., Tong, G.Q., Lim, C.A., Robson, P., Zhong, S., and Ng, H.H. (2008). A core Klf circuitry regulates self-renewal of embryonic stem cells. Nat. Cell Biol. 10, 353-360.

Kelly, T.J., Brümmer, A., Hooshdaran, N., Tariveranmoshabad, M., and Zamudio, J.R. (2019). Temporal Control of the TGF- $\beta$ Signaling Network by Mouse ESC MicroRNA Targets of Different Affinities. Cell Rep. 29, 2702-2717.e7.

Kennerdell, J.R., Liu, N., and Bonini, N.M. (2018). MiR-34 inhibits polycomb repressive complex 2 to modulate chaperone expression and promote healthy brain aging. Nat. Commun. 9.

Kent, W.J., Zweig, A.S., Barber, G., Hinrichs, A.S., and Karolchik, D. (2010). BigWig and BigBed: Enabling browsing of large distributed datasets. Bioinformatics 26, 2204-2207.

Kulakovskiy, I. V, Vorontsov, I.E., Yevshin, I.S., Sharipov, R.N., Fedorova, A.D., Rumynskiy, E.I., Medvedeva, Y.A., Magana-Mora, A., Bajic, V.B., Papatsenko, D.A., et al. (2018). HOCOMOCO: Towards a complete collection of transcription factor binding models for human and mouse via largescale ChIP-Seq analysis. Nucleic Acids Res. 46, D252-D259.

Langmead, B., and Salzberg, S.L. (2012). Fast gapped-read alignment with Bowtie 2. Nat. Methods 201294 9, 357-359.

Larsson, J., Diagrams, P.G.-S., and 2018, undefined (2018). A Case Study in Fitting AreaProportional Euler Diagrams with Ellipses using eulerr. Researchgate.Net.

Li, H., Handsaker, B., Wysoker, A., Fennell, T., Ruan, J., Homer, N., Marth, G., Abecasis, G., and Durbin, R. (2009). The Sequence Alignment/Map format and SAMtools. Bioinformatics 25, 20782079.

Li, X., Wang, X., Cheng, Z., and Zhu, Q. (2020). AGO2 and its partners: a silencing complex, a chromatin modulator, and new features. Crit. Rev. Biochem. Mol. Biol. 55, 33-53.

Liao, Y., Smyth, G.K., and Shi, W. (2014). FeatureCounts: An efficient general purpose program for assigning sequence reads to genomic features. Bioinformatics 30, 923-930.

Love, M.I., Huber, W., and Anders, S. (2014). Moderated estimation of fold change and dispersion for 
RNA-seq data with DESeq2. Genome Biol. 15, 550.

Lun, A.T.L., and Smyth, G.K. (2016). csaw: a Bioconductor package for differential binding analysis of ChIP-seq data using sliding windows. Nucleic Acids Res. 44, e45.

Lykke-Andersen, K., Gilchrist, M.J., Grabarek, J.B., Das, P., Miska, E., and Zernicka-Goetz, M. (2008). Maternal Argonaute 2 Is Essential for Early Mouse Development at the Maternal-Zygotic Transition. Mol. Biol. Cell 19, 4383-4392.

Margueron, R., and Reinberg, D. (2011). The Polycomb complex PRC2 and its mark in life. Nature 469, 343-349.

Martin, M. (2011). Cutadapt removes adapter sequences from high-throughput sequencing reads. EMBnet.Journal 17, 10-12.

McKinney, W. (2010). Data Structures for Statistical Computing in Python. Proc. 9th Python Sci. Conf. $1,56-61$.

Meister, G. (2013). Argonaute proteins: Functional insights and emerging roles. Nat. Rev. Genet. 14, 447-459.

Moshkovich, N., Nisha, P., Boyle, P.J., Thompson, B.A., Dale, R.K., and Lei, E.P. (2011). RNAiindependent role for argonaute2 in CTCF/CP190 chromatin insulator function. Genes Dev. 25, 16861701.

Müller, M., Fazi, F., and Ciaudo, C. (2020). Argonaute Proteins: From Structure to Function in Development and Pathological Cell Fate Determination. Front. Cell Dev. Biol. 7, 1-10.

Müller, M., Fäh, T., Schäfer, M., Luitz, J., Stalder, P., Arora, R., Ngondo, R.P., and Ciaudo, C. (2021). AGO1 regulates major satellite transcripts and $\mathrm{H} 3 \mathrm{~K} 9 \mathrm{me} 3$ distribution at pericentromeric regions in mESCs. BioRxiv 1-45.

Ngondo, R.P., Cirera-Salinas, D., Yu, J., Wischnewski, H., Bodak, M., Vandormael-Pournin, S., Geiselmann, A., Wettstein, R., Luitz, J., Cohen-Tannoudji, M., et al. (2018). Argonaute 2 Is Required for Extra-embryonic Endoderm Differentiation of Mouse Embryonic Stem Cells. Stem Cell Reports 10, 461-476.

Nora, E.P., Goloborodko, A., Valton, A.L., Gibcus, J.H., Uebersohn, A., Abdennur, N., Dekker, J., Mirny, L.A., and Bruneau, B.G. (2017). Targeted Degradation of CTCF Decouples Local Insulation of Chromosome Domains from Genomic Compartmentalization. Cell 169, 930-944.e22.

Oudelaar, A.M., and Higgs, D.R. (2021). The relationship between genome structure and function. Nat. Rev. Genet. 22, 154-168.

Quinlan, A.R., and Hall, I.M. (2010). BEDTools: A flexible suite of utilities for comparing genomic features. Bioinformatics 26, 841-842.

Ramírez, F., Ryan, D.P., Grüning, B., Bhardwaj, V., Kilpert, F., Richter, A.S., Heyne, S., Dündar, F., and Manke, T. (2016). deepTools2: a next generation web server for deep-sequencing data analysis. Nucleic Acids Res. 44, W160-W165.

Ramírez, F., Bhardwaj, V., Arrigoni, L., Lam, K.C., Grüning, B.A., Villaveces, J., Habermann, B., Akhtar, A., and Manke, T. (2018). High-resolution TADs reveal DNA sequences underlying genome organization in flies. Nat. Commun. 9.

Reske, J.J., Wilson, M.R., and Chandler, R.L. (2020). ATAC-seq normalization method can significantly affect differential accessibility analysis and interpretation. Epigenetics Chromatin 2020 $13113,1-17$.

Sarshad, A.A., Juan, A.H., Muler, A.I.C., Anastasakis, D.G., Wang, X., Genzor, P., Feng, X., Tsai, P.F., Sun, H.-W., Haase, A.D., et al. (2018). Argonaute-miRNA Complexes Silence Target mRNAs in the Nucleus of Mammalian Stem Cells. Mol. Cell 71, 1040-1050.e8. 
Schäfer, M., Nabih, A., Spies, D., Bodak, M., Stalder, P., Ngondo, R.P., Liechti, L.A., Sajic, T., Aebersold, R., Gatfield, D., et al. (2021). Integrative analysis allows a global and precise identification of functional miRNA target genes in mESCs. BioRxiv.

Shannon, P., Markiel, A., Ozier, O., Baliga, N.S., Wang, J.T., Ramage, D., Amin, N., Schwikowski, B., and Ideker, T. (2003). Cytoscape: A Software Environment for Integrated Models. Genome Res. 13, 2498-2504.

Shivram, H., Le, S. V., and lyer, V.R. (2019). MicroRNAs reinforce repression of PRC2 transcriptional targets independently and through a feed-forward regulatory network. Genome Res. 29, 184-192.

Shuaib, M., Parsi, K.M., Thimma, M., Adroub, S.A., Kawaji, H., Seridi, L., Ghosheh, Y., Fort, A., Fallatah, B., Ravasi, T., et al. (2019). Nuclear AGO1 Regulates Gene Expression by Affecting Chromatin Architecture in Human Cells. Cell Syst. 9, 446-458.e6.

Smibert, P., Yang, J.S., Azzam, G., Liu, J.L., and Lai, E.C. (2013). Homeostatic control of Argonaute stability by microRNA availability. Nat. Struct. Mol. Biol. 20, 789-795.

Spitz, F., and Furlong, E.E.M. (2012). Transcription factors: From enhancer binding to developmental control. Nat. Rev. Genet. 13, 613-626.

Waskom, M. (2021). Seaborn: Statistical Data Visualization. J. Open Source Softw. 6, 3021. 


\section{Figure \& Figure Legends}

A

\section{Intersection of DEGs in} RNAi_KO mESCs

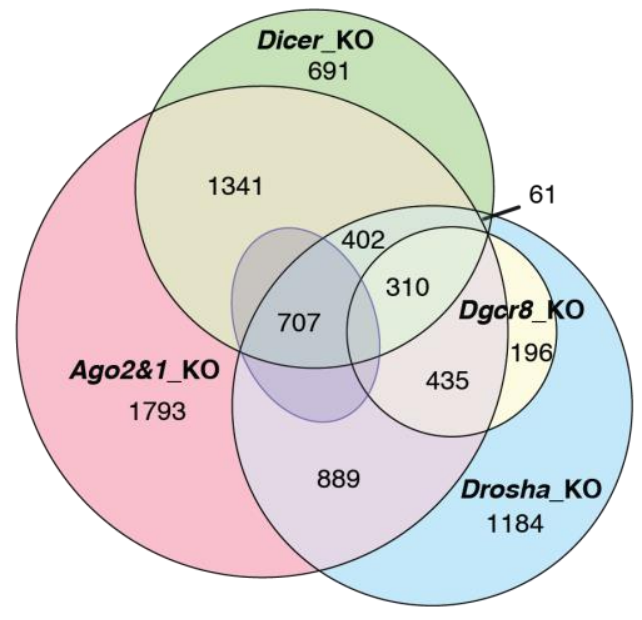

C GO Analysis Ago2\&1_KO specific DEGs

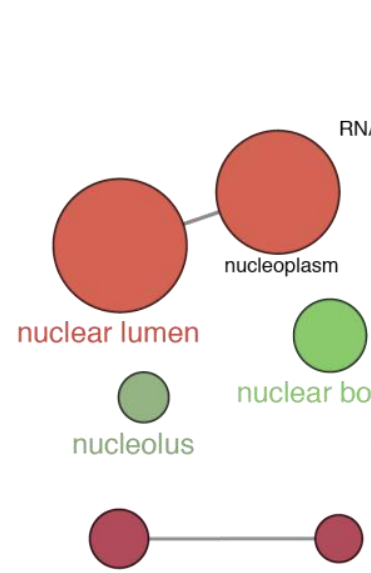

microtubule cytoskeleton

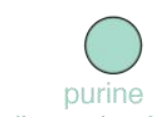

ribonucleotide

binding

positive regulation of macromolecule biosynthetic process microtubule organizing center

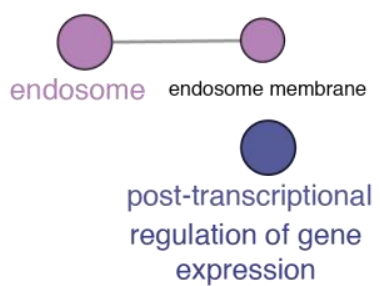

Ago2\&1_KO Dgcr8_KO

- Dicer_KO

Drosha_KO

- miRNA targets as identified by Schäfer et al., 2021
B

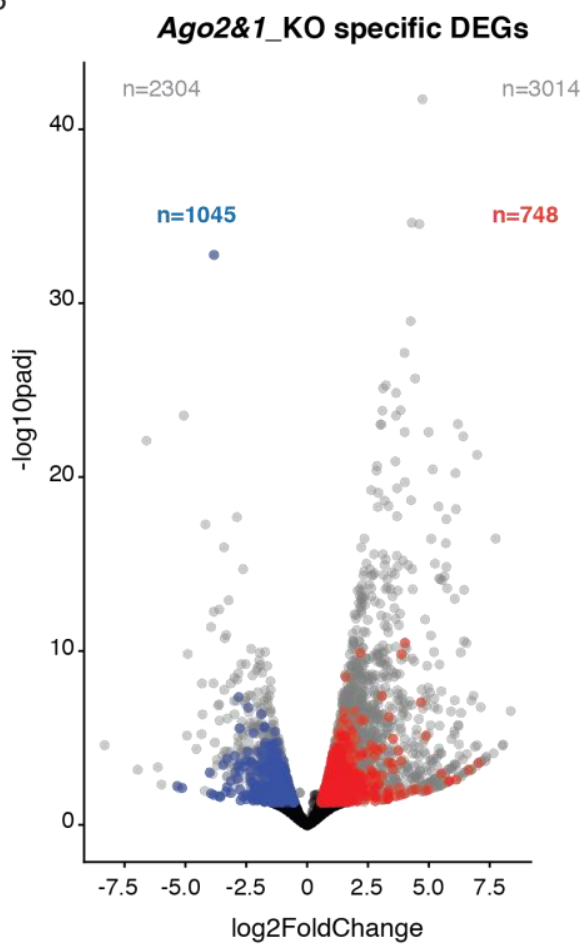

RNA metabolic

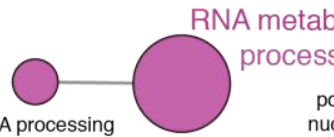

positive regulation of

nucleobase-containing compund metabolic process

regulation of RNA metabolic process
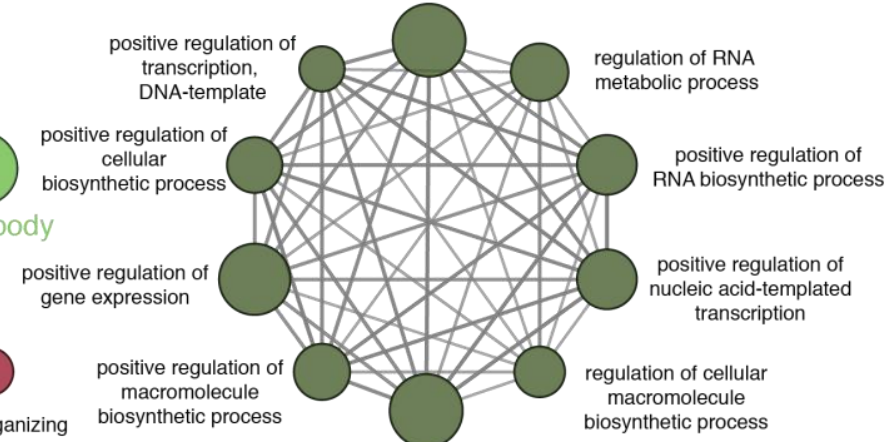

positive regulation of RNA metabolic process

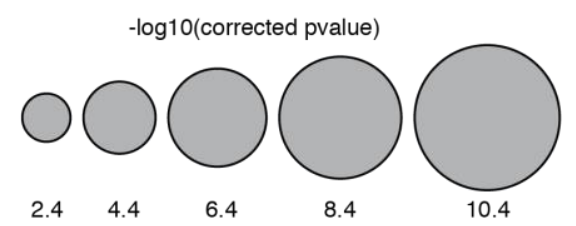

Figure 1. Ago2\&1 KO mESCs display a distinct transcriptomic profile

(A) Venn diagram representing the overlap of differentially expressed genes (DEGs) from different miRNA_KO mESCs (Dgcr8_KO, Drosha_KO, Dicer_KO, Ago2\&1_KO) and the 707 miRNA targets from (Schäfer et al., 2021). Numbers indicate the gene set sizes of different overlaps. Ago2\&1_KO mESCs have 1793 specific DEGs (Dataset EV1). 
(B) Volcano plot showing the Ago2\&1_KO DEGs and the 1793 specific DEGs. The full set of Ago2\&1_KO DEGs is shown in gray. Highlighted in red are the Ago2\&1_KO specific DEGs that are upregulated (748) and in blue the Ago2\&1_KO specific DEGs that are downregulated (1045).

(C) Gene Ontology analysis on the Ago2\&1_KO the 1793 specific DEGs. The GO analysis has been performed with ClueGO (Bindea et al., 2009). The size of the circles corresponds to their p-value. 
A

RNA-seq Ago1_KO vs. WT

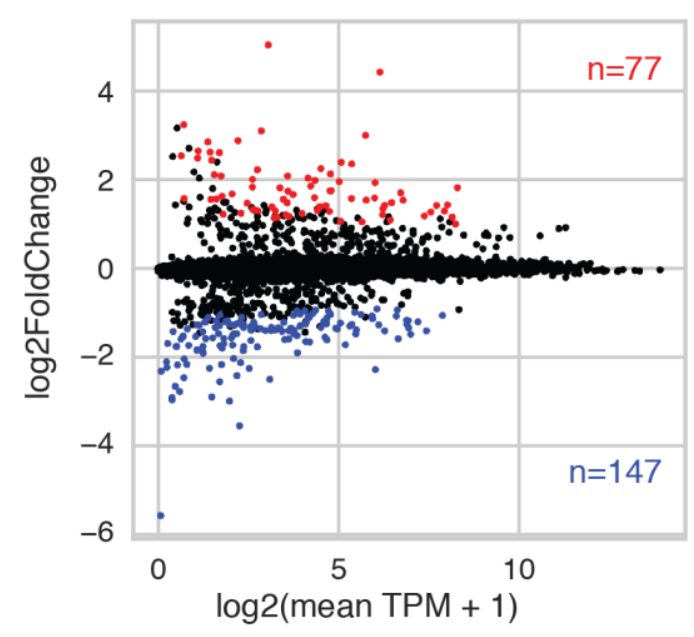

B

RNA-seq Ago2_KO vs. WT

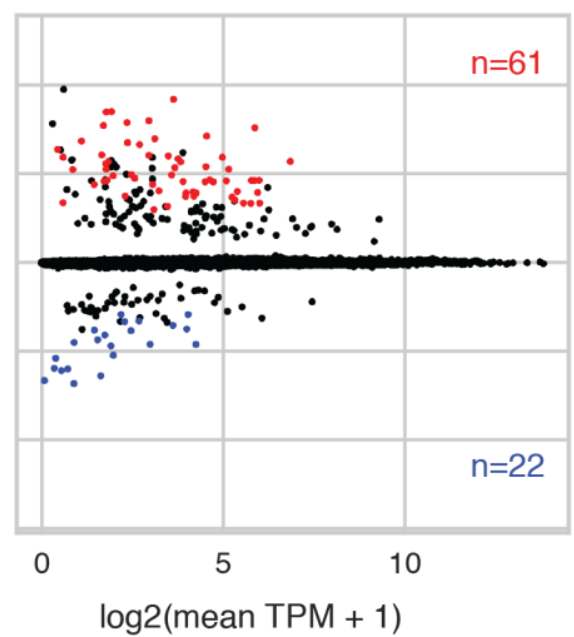

C

\section{Differentially expressed genes}

(DEGs) in Ago_KO mESCs

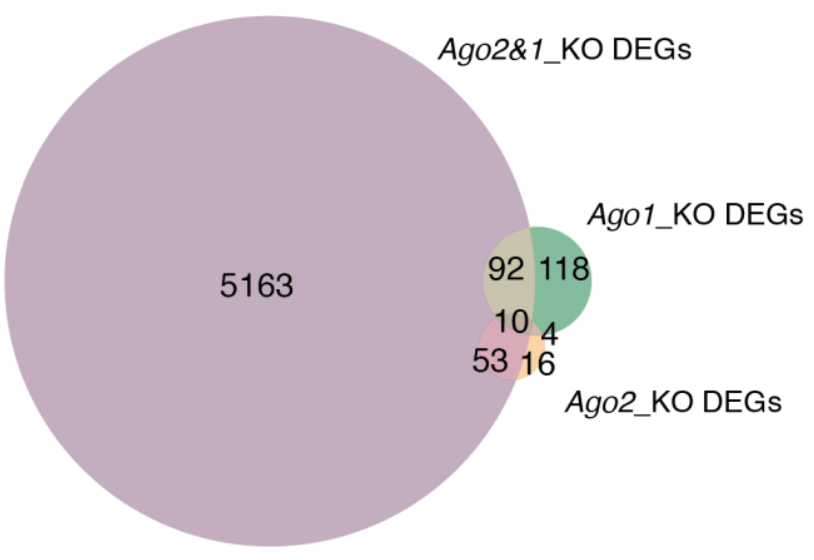

Figure EV1. Ago_KO transcriptomic analysis

(A, B) MA plots showing the differential gene expression for the Ago1_KO mESCs versus WT as in (Müller et al., 2021) (A) and Ago2_KO vs WT as in (Ngondo et al., 2018) (B). Highlighted in red are the statistically significant upregulated and in blue the statistically significant downregulated genes. The number of genes in those groups are denoted in the right corners (Dataset EV1). TPM: transcripts per Million.

(C) Venn diagram showing the overlap between differentially expressed genes (DEGs) in Ago2\&1_KO, Ago1_KO and Ago2_KO mESCs. Notably, Ago1_KO and Ago2_KO show comparably low DEGs and overlap only partially with Ago2\&1_KO. 
A

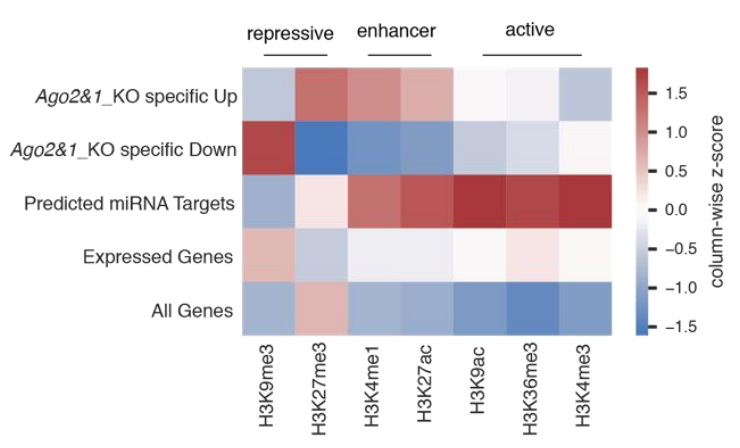

B

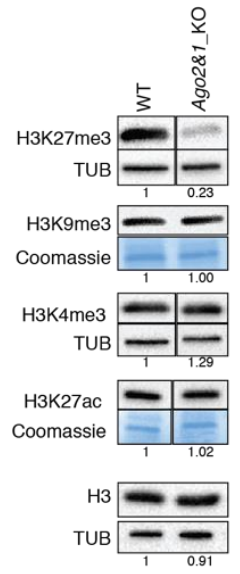

C

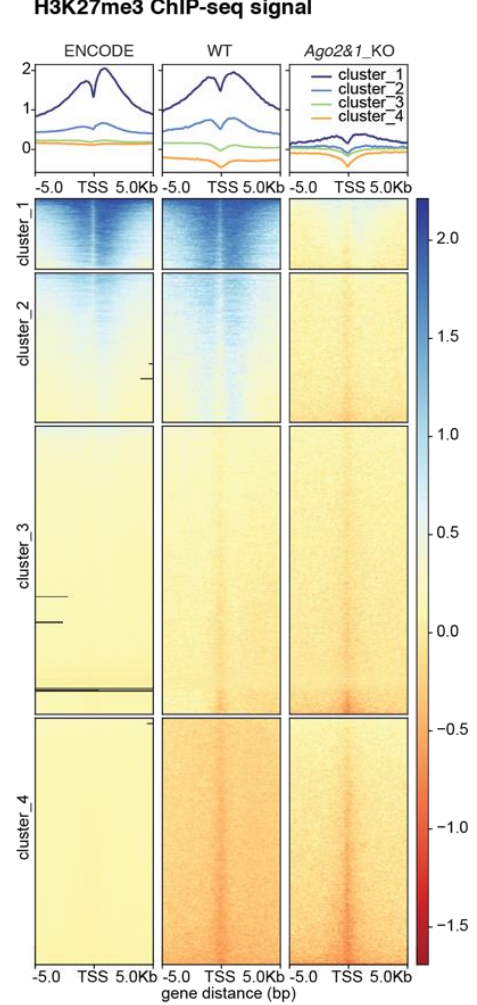

-5.0 TŚS $5.0 \mathrm{~Kb}-5.0$ TŚs $5.0 \mathrm{~kb}$ - 50

D H3K27me3 ChIP-seq comparison

\begin{tabular}{lccccc}
\hline 180.2 & 180.4 & 180.6 & 180.8 & 181 & Mbp \\
& & chr1 & & &
\end{tabular}

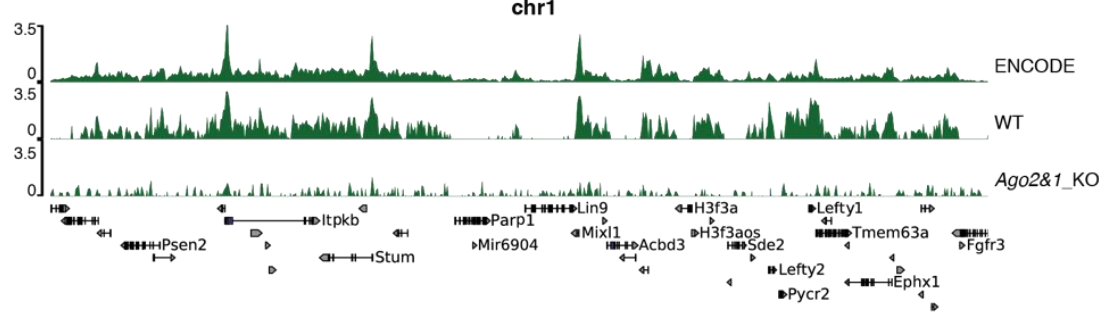

E ChIP-seq and RNA-seq integration

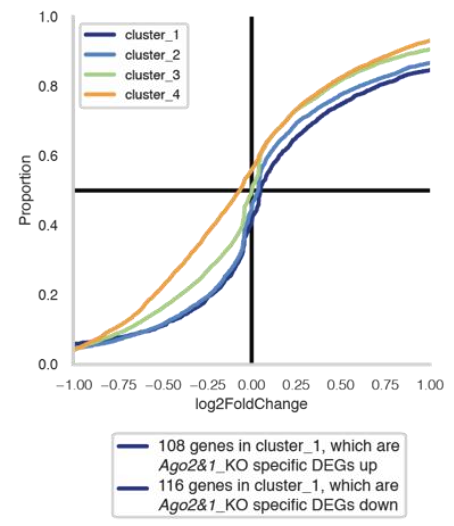

F
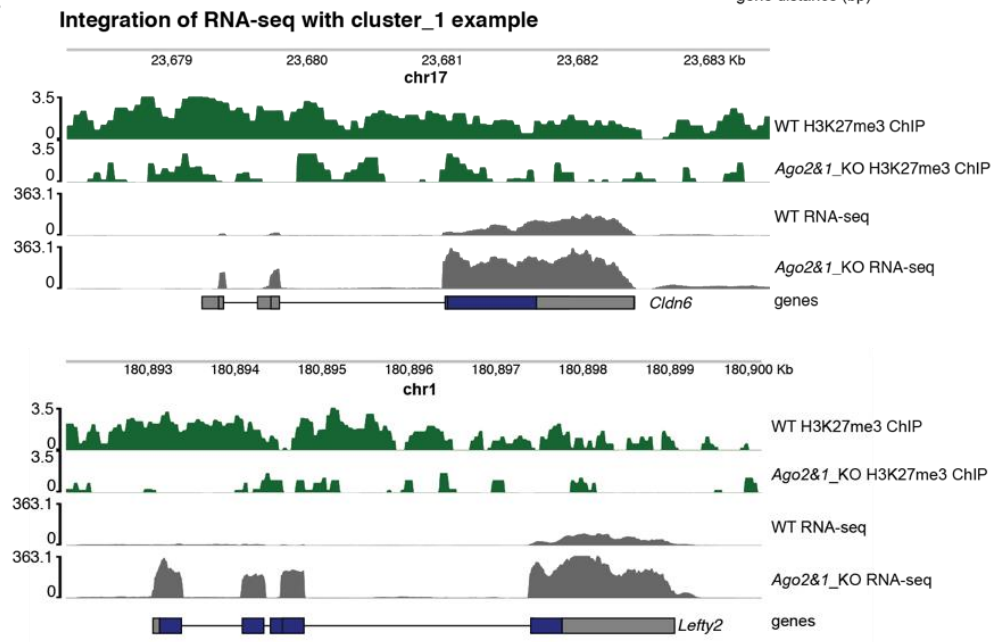

Figure 2. Integration of the Ago2\&1_KO transcriptome with histone modification datasets

(A) Heatmap showing average histone modification signals at gene regions as derived from ENCODE datasets (see Materials \& Methods) for five different gene groups; Ago2\&1_KO specific up- and downregulated DEGs, 707 predicted functional miRNA target genes from (Schäfer et al., 2021), all expressed genes in mESCs and all annotated genes in mESCs. Histone marks were annotated with their previously described predominant functions (Bannister and Kouzarides, 2011). Repressive histone marks: H3K27me3 and H3K9me3, enhancer marks: H3K4me1 and H3K27ac, activating marks: H3K36me3 and H3K4me3. Columns were individually z-score normalized.

(B) Representative Western blots for H3K27me3, H3K9me3, H3K4me3, H3K27ac and H3 in WT and Ago2\&1_KO mESCs out of $n=3$ independent experiments. Tubulin (TUB) and Coomassie were used as a loading control. Quantification for each individual experiment is shown below the blot. 
(C) H3K27me3 ChIP-seq heatmaps of the transcription start sites (TSS) for the full set of annotated mouse transcripts. Shown is one replicate per condition/experiment for ENCODE WT (Davis et al., 2018; Dunham et al., 2012), WT and Ago2\&1_KO samples (see Materials \& Methods). The shown transcript regions have been divided into four different clusters using k-means clustering (Dataset EV2). Shown are $+/-5 \mathrm{~kb}$ from the transcriptional start site (TSS).

(D) Genome browser view of a region derived from transcripts from cluster_1. ChIP-seq coverage signals are shown for the three samples from (C) along with annotated genes in that region (bottom).

(E) Cumulative Distribution Function (CDF) plot showing the differential expression in Ago2\&1_KO versus WT of genes associated with the different ChIP peak clusters identified in the ENCODE and WT datasets. The x-axis represents the log2FoldChange of the Ago2\&1_KO versus WT RNA-seq and the $y$-axis the cumulative proportion over the full set of log2FoldChanges for each cluster.

(F) Genome browser view of two example genes from cluster_1 (Cldn6 and Lefty2) showing both ChIP-seq and RNA-seq coverages for WT and Ago2\&1_KO (one sample per experiment and condition). The $y$-axis represents the deposition of H3K27me3 (for the ChIP-seq in green) or the expression levels of the RNA-seq in gray. 
A

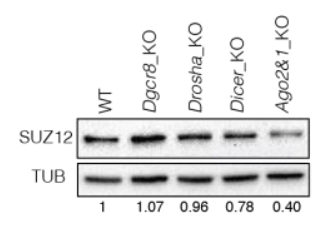

$\mathrm{B}$

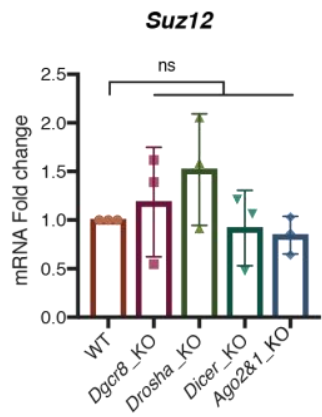

E

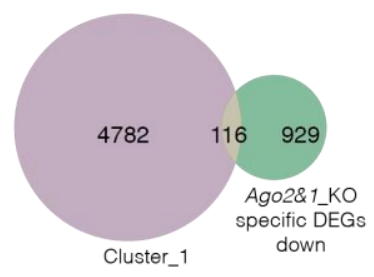

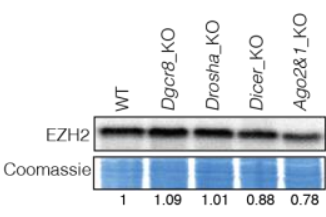

Ezh2

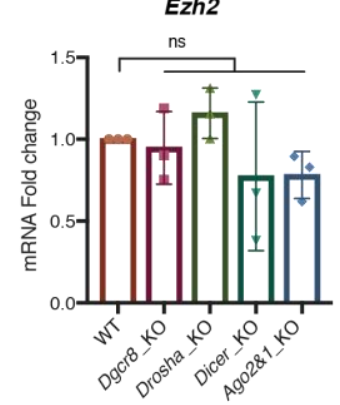

C

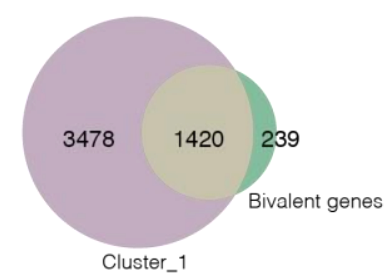

$\mathrm{D}$

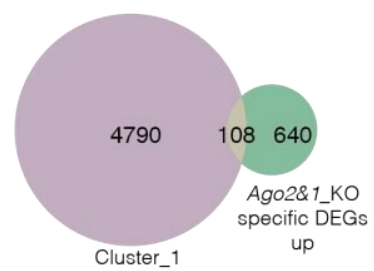

$\mathrm{F}$

Integration of RNA-seq with cluster_3 example

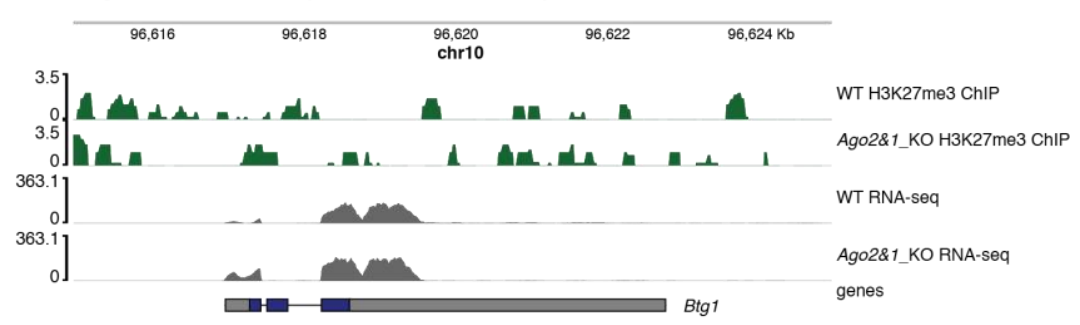

Figure EV2. Expression of Suz12 and Ezh2 PRC2 members in Ago2\&1_KO mESCs and the integration of the Ago2\&1_KO transcriptome with the H3K27me3 ChIP-seq

(A) Representative Western blots for SUZ12 and EZH2 in WT, Dgcr8_KO, Drosha_KO, Dicer_KO and Ago2\&1_KO mESCs out of $\mathrm{n}=3$ independent experiments. Tubulin (TUB) and Coomassie were used as a loading control. Quantification for each individual experiment is shown below the blot.

(B) Quantitative-RT-PCR results for Suz12 and Ezh2 in WT, Dgcr8_KO, Drosha_KO,Dicer_KO and Ago2\&1_KO, from $\mathrm{n}=3$ independent experiments. $\mathrm{ns}=$ non-significant, unpaired t-test.

(C) Venn diagram showing the overlap of genes in the ChIP-seq cluster_1 with high-confidence bivalent genes, identified by (Asenjo et al., 2020).

(D, E) Venn diagrams showing the overlap of genes in the ChIP-seq cluster_1 with Ago2\&1_KO specific up (D) and down (E) DEGs.

(F) Genome browser view of an example gene from the ChIP-seq cluster_3 (Btg1), showing both ChIP-seq and RNA-seq coverages. Depicted are genome tracks for the WT and Ago2\&1_KO ChIPseq (one sample per experiment and condition). The $y$-axis represents the deposition of H3K27me3 (for the ChIP-seq in green) or the expression levels of the RNA-seq in gray. 
bioRxiv preprint doi: https://doi.org/10.1101/2021.1018.464771: this version posted October 19, 2021. The copyright holder for this preprint (which was not certified by peer review) is the author/funder, who has granted bioRxiv a license to display the preprint in perpetuity. It is made available under aCC-BY-NC-ND 4.0 International license.

A Chromatin accessibility B
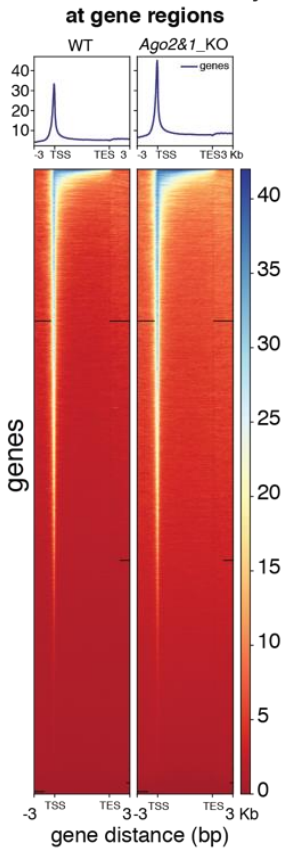

$\mathrm{E}$ Integration of diffTF identified targets with RNA-seq at promoters
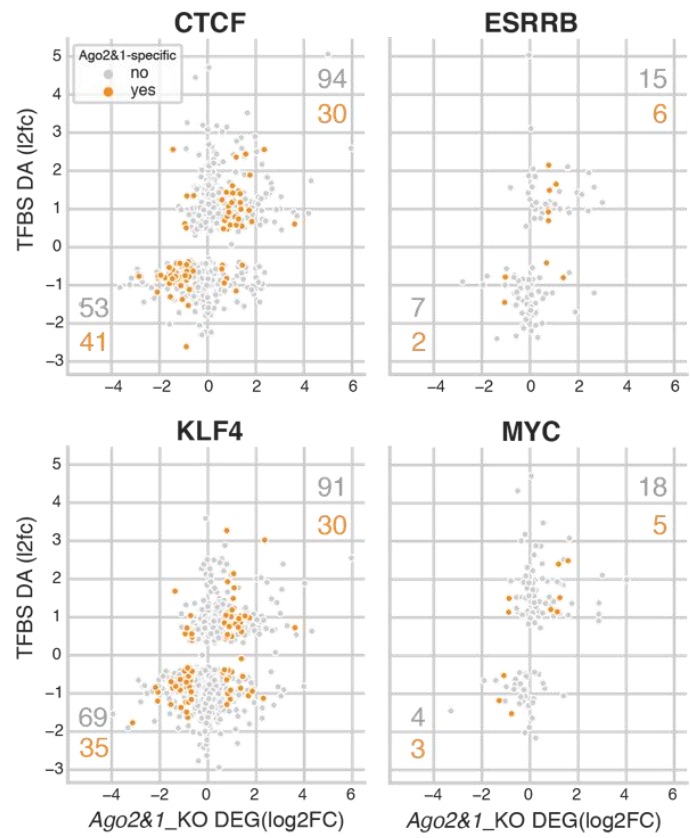

REST

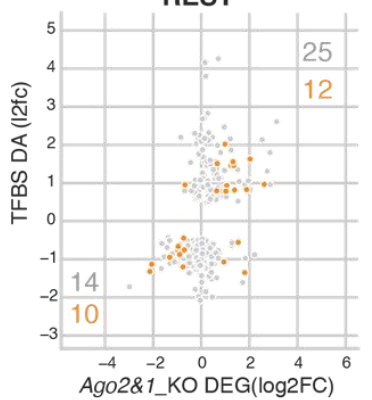

D

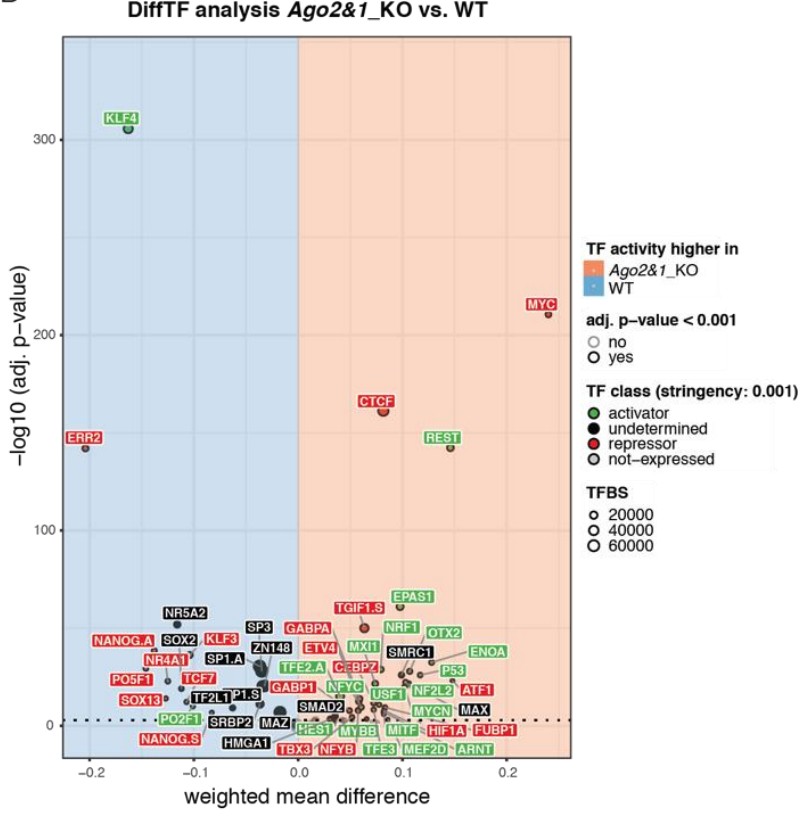

F Integration of diffTF identified targets with RNA-seq at enhancers
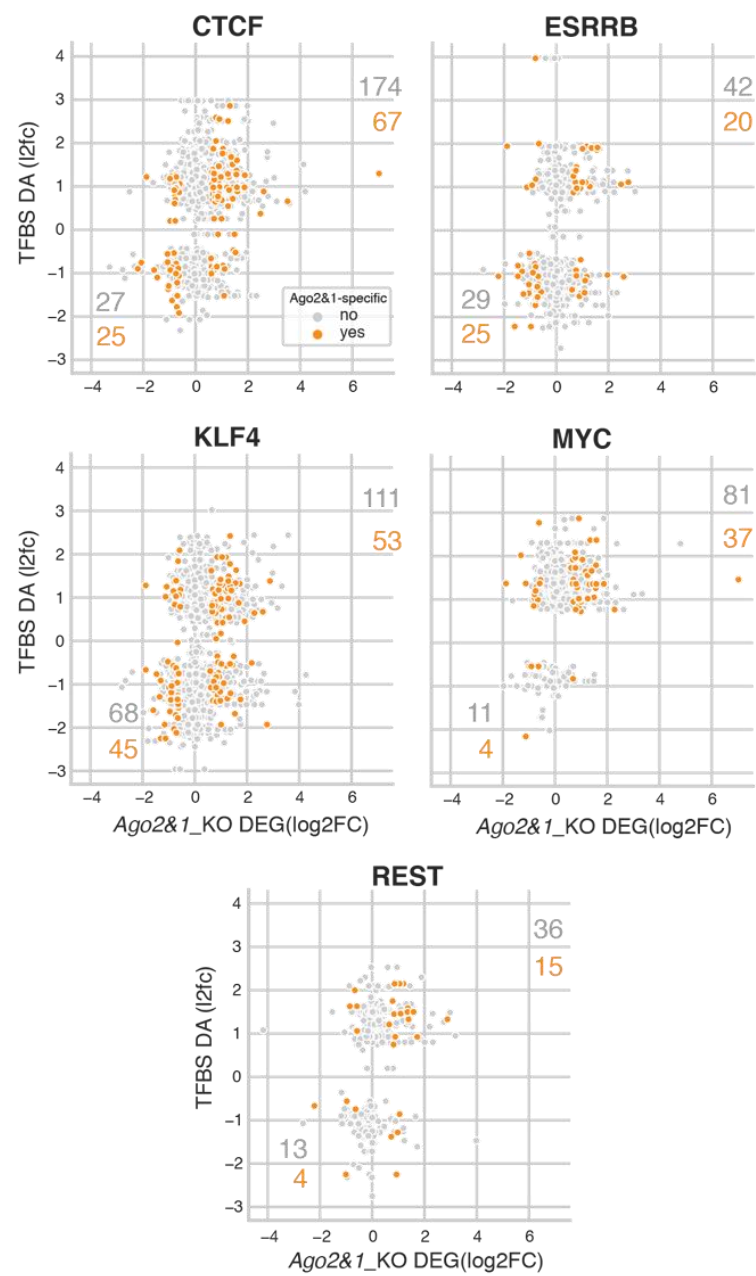

Figure 3. Integration of the Ago2\&1_KO transcriptome with chromatin accessibility and TF binding 
(A) Heatmap and profile plots for WT and Ago2\&1_KO chromatin accessibility as assessed by ATACseq. Transcription start sites (TSS) to transcription end sites (TES) with 3kbp margins are shown for the full set of annotated transcripts. Representative samples of biological duplicates are shown.

$(B, C)$ Average signal of chromatin accessibility at TSS/promoter regions (B) and enhancer regions (C), as annotated by (González-Ramírez et al., 2021) for WT and Ago2\&1_KO samples as assessed by ATAC-seq.

(D) Volcano plot of differential chromatin accessibility (DA) at transcription factor (TF) binding sites (BS) for 88 expressed TFs as computed by diffTF (Berest et al., 2019) (Dataset EV4). x-axis shows the difference in chromatin accessibility between Ago2\&1_KO and WT ATAC-seq samples, where the red area denotes an increase in chromatin accessibility in the Ago2\&1_KO samples and the blue area a decrease. TFs are annotated as activators (green) or repressors (red) according to the DA at their binding sites and their expression levels, based on RNA-seq data.

(E, F) Scatterplots showing differential expression (RNA-seq) versus DA of potential target genes associated with TFBS from (D) for the five TFs with most significant DA binding sites from (D). TFBS were associated with genes by promoter proximity (E, $<1 \mathrm{kbp}$ distance to TSS) or enhancer proximity (F) (González-Ramírez et al., 2021). Genes are denoted in orange if they are Ago2\&1_KO specific DEGs. 
bioRxiv preprint doi: https//doi.org/10.1101/2021.10.18.464771; this version posted October 19,2021 . The copyright holder for this preprint (which was not certified by peer review) is the author/funder, who has granted bioRxiv a license to display the preprint in perpetuity. It is made available under aCC-BY-NC-ND 4.0 International license.

A

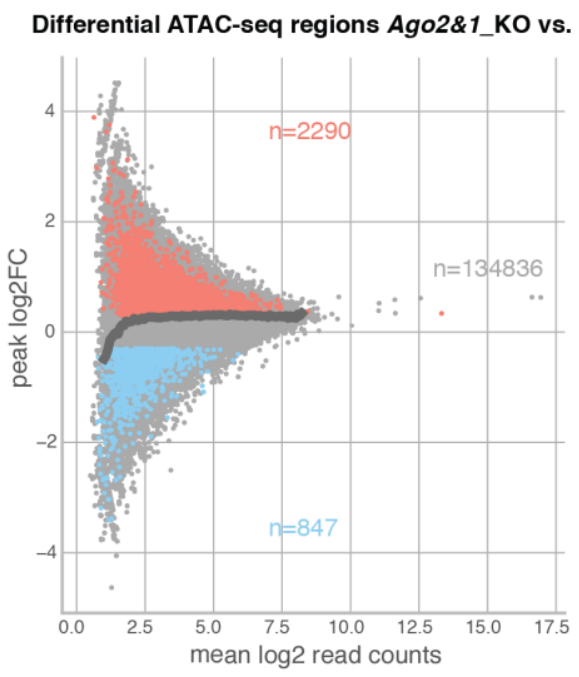

C

Differential ATAC-seq regions Ago2_KO vs.WT

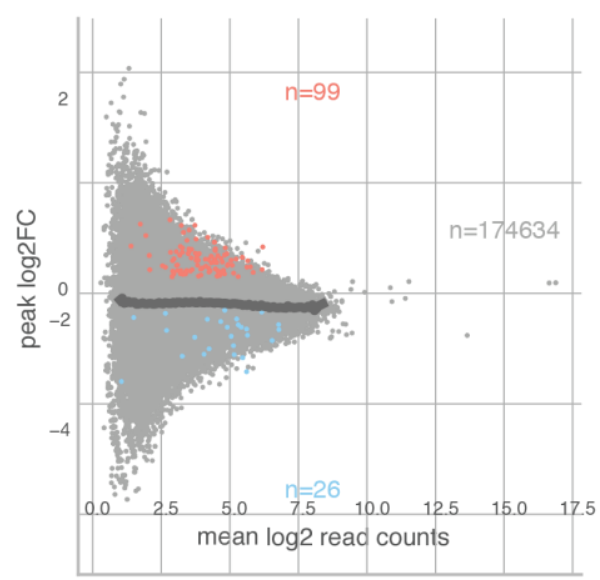

E

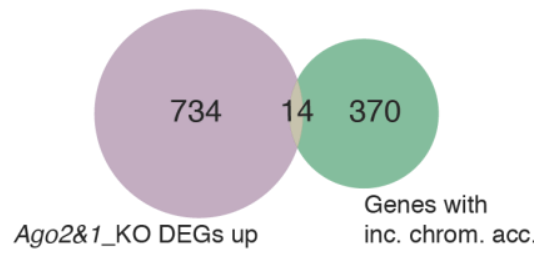

B

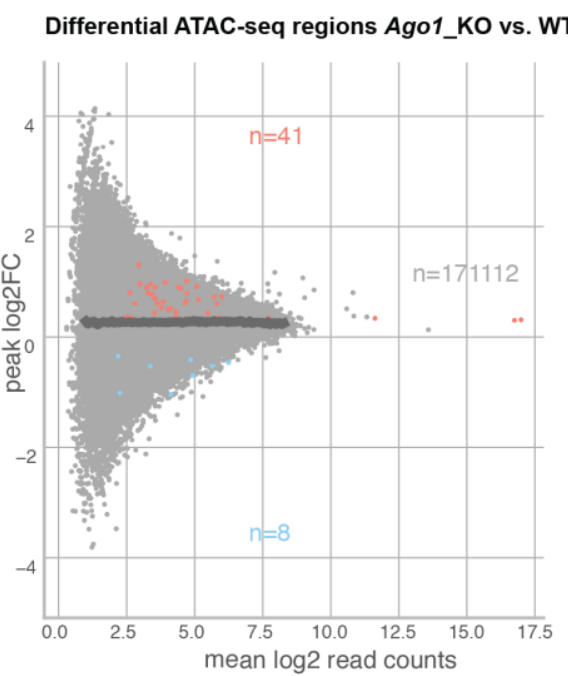

D

Integration of differential ATAC-seq regions at gene promoters with Ago2\&1_KO DEGs

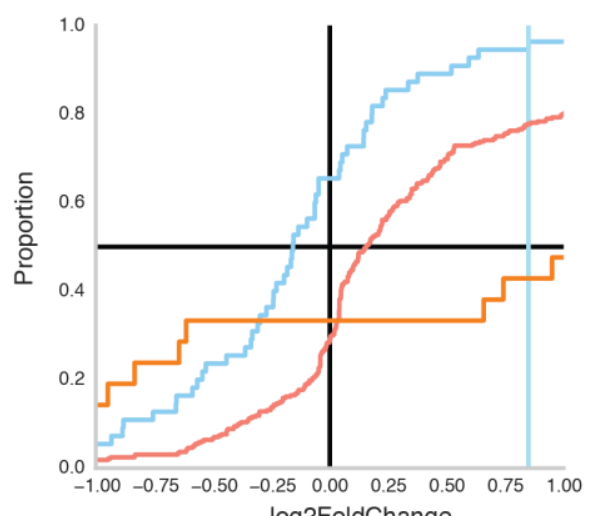

log2FoldChange

76 genes with dec. chrom. access. at promoter (<1000bp. DA Pval<0.05) -1 genes with dec. chrom. access. are Ago2\&1_KO specific DEGs 384 genes with inc. chrom. access. at promoter (<1000bp, DA Pval<0.05) 21 genes with inc. chrom. access. are Ago2\&1_KO specific DEGs

$\mathrm{F}$

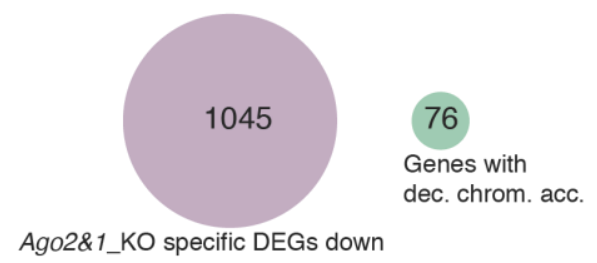

Figure EV3. Integration of the Agos_KO transcriptome with chromatin accessibility

(A, B, C) MA plots of genomic regions with detectable chromatin accessibility (based on Genrich peak calling). Differential accessibility (DA) at those regions was assessed using CSAW/EdgeR for Ago2\&1_KO vs WT (A), Ago1_KO vs WT (B) Ago2_KO vs WT (C). Regions with an absolute log2FC $>0.3$ and adjusted $p$-value $<0.05$ were considered as statistically significant and are colored (red and blue) and counted.

(D) Cumulative Distribution Function (CDF) plot of differential expression (DE, log2FoldChange of RNA-seq Ago2\&1_KO vs WT) for genes with promoters in proximity (<1000bp) of differentially 
accessible regions ( $p$-value $<0.05$ ) with increased (blue) or decreased (red) accessibility. DEGs were additionally shown after filtering by Ago2\&1_KO specific DEGs.

(E, F) Overlap of genes associated with differential accessibility (as in (D)) with Ago2\&1_KO specific up (E) and down (F) DEGs. 
bioRxiv preprint doi: https//doi.org/10,1101/2021.10.18.464771; this version posted October 19,2021 . The copyright holder for this preprint (which was not certified by peer review) is the author/funder, who has granted bioRxiv a license to display the preprint in perpetuity. It is made available under aCC-BY-NC-ND 4.0 International license.
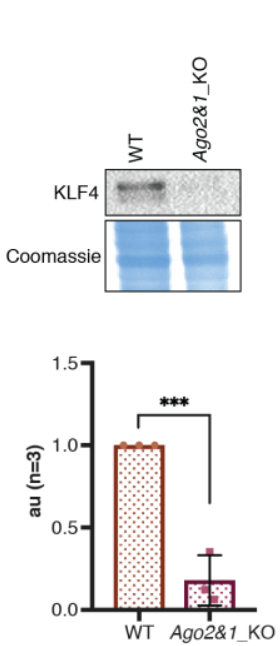

Overlap of CTCF-ChIP-seq-associated
C

Overlap of KLF4-ChIP-seq-peak associated genes with Ago2\&1_KO specific DEGs
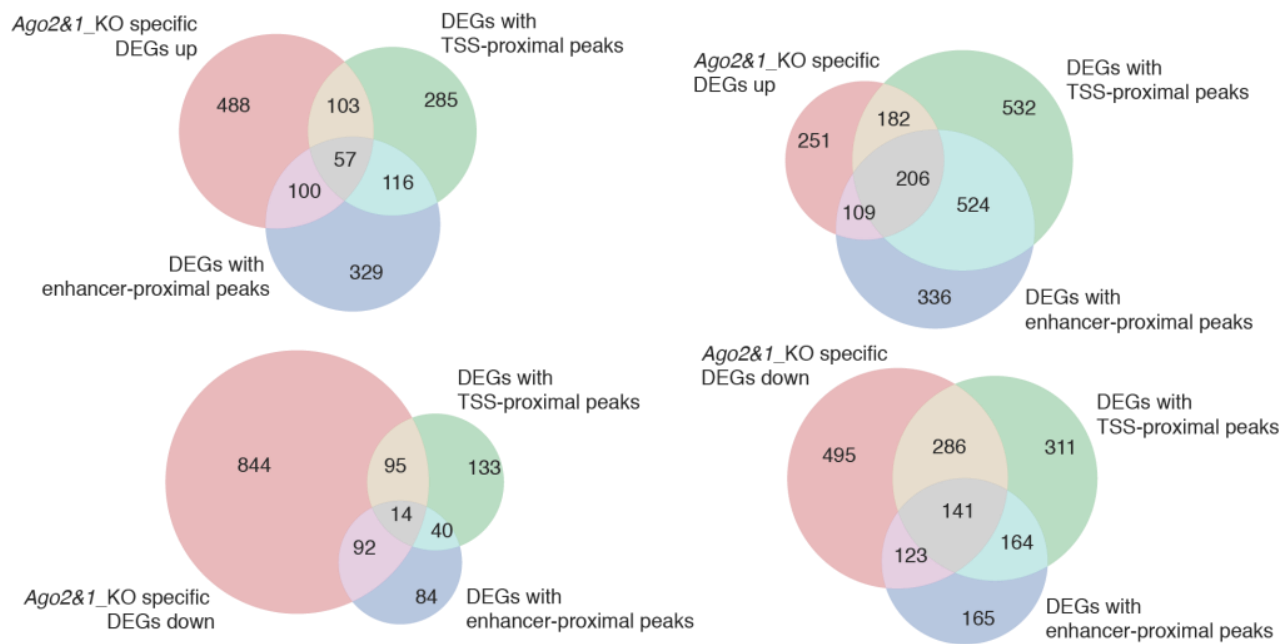

D

Ago2\&1_KO specific DEGs up explained

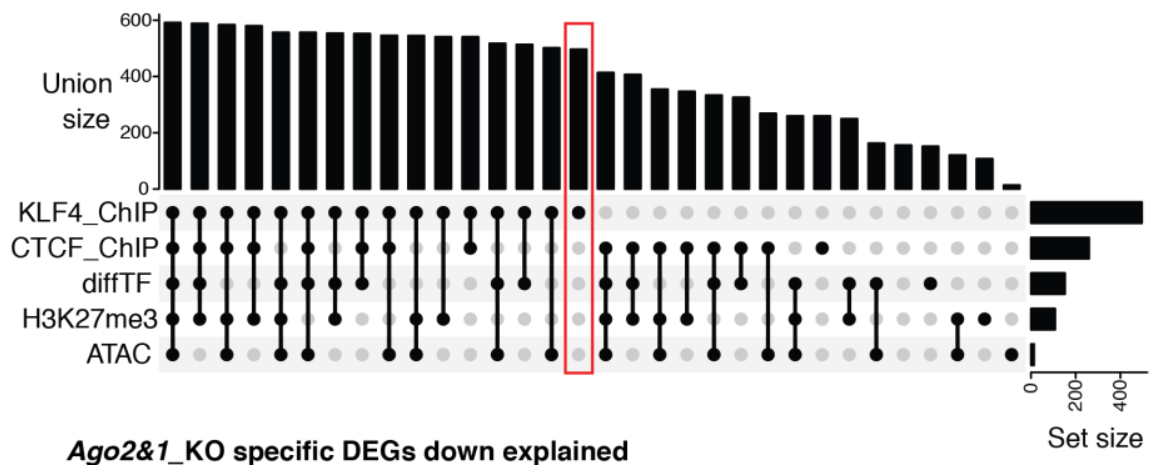

$\mathrm{E}$

Ago2\&1_KO specific DEGs down explained

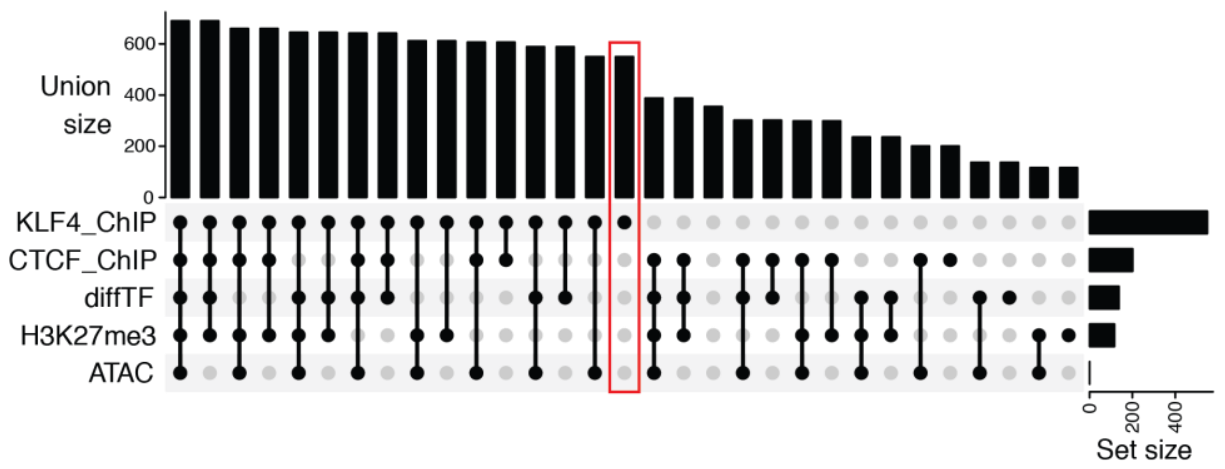

Figure 4. Identification of KLF4-/CTCF-targets and complete integration

(A) Representative Western blot (top) for KLF4 in WT and Ago2\&1_KO mESCs and quantification (bottom) out of $n=3$ independent experiments, ${ }^{* *} \mathrm{p}<0.001$, unpaired t-test.

$(B, C)$ Venn diagrams of genes identified by CTCF (B) and KLF4 (C) ChIP-seq peak analysis with Ago2\&1_KO specific DEGs. ChIP-seq peaks were associated with genes by promoter proximity (transcription start site (TSS) < 1000bp, green circle) and by enhancer-proximity (overlap with annotated enhancers by (González-Ramírez et al., 2021)). Different Venn diagrams are shown for 
upregulated (top) and downregulated (bottom) gene sets and only statistically significant Ago2\&1_KO differentially expressed genes (DEGs) are considered.

(D, E) UpSet plots showing the number Ago2\&1_KO specific upregulated (D) and downregulated (E) genes explained by one or multiple combined regulatory mechanisms as studied in this paper (Dataset EV5). The dot-connected lines indicate which gene explanation sets were combined and the bar above denotes the total number of genes explained by that combination. The bars on the right indicate the total number of explained genes explained by each individual analysis (these are thus redundant with the columns representing a single dot). Notably, KLF4 ChIP-seq analysis (red box, and top-most bar on the right) alone already explained the majority of all explained genes. 

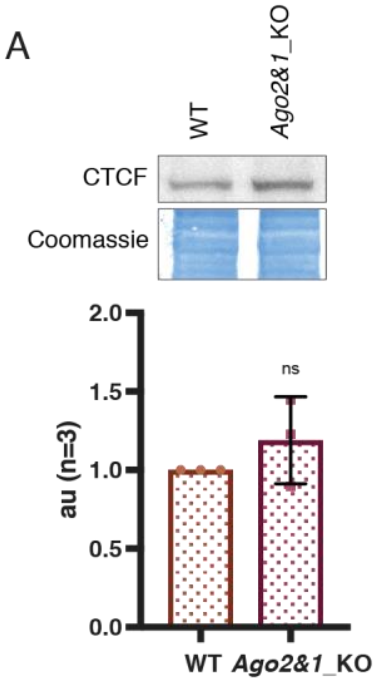

B

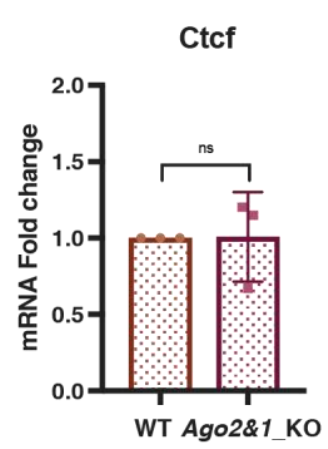

C

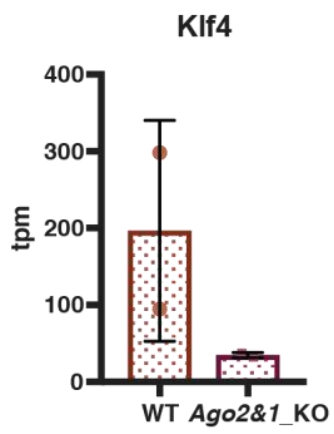

Figure EV4. Characterization of CTCF and KLF4 in mESCs.

(A) Representative Western blot (top) for CTCF in WT and Ago2\&1_KO mESCs and quantification (bottom) out of $\mathrm{n}=3$ independent experiments, $\mathrm{ns}=\mathrm{not}$ significant, unpaired t-test.

(B) Ctcf qRT-PCR results in WT and Ago2\&1_KO mESCs. n=3 independent experiments. ns=not significant, unpaired t-test.

(C) Klf4 tpm counts from the RNA-seq data (Dataset EV1) in WT and Ago2\&1_KO mESCs. 